Research Articles: Systems/Circuits

\title{
The sensory and motor components of the cortical hierarchy are coupled to the rhythm of the stomach during rest
}

https://doi.org/10.1523/JNEUROSCI.1285-21.2021

Cite as: J. Neurosci 2022; 10.1523/JNEUROSCI.1285-21.2021

Received: 22 June 2021

Revised: 30 November 2021

Accepted: 17 December 2021

This Early Release article has been peer-reviewed and accepted, but has not been through the composition and copyediting processes. The final version may differ slightly in style or formatting and will contain links to any extended data.

Alerts: Sign up at www.jneurosci.org/alerts to receive customized email alerts when the fully formatted version of this article is published.

Copyright @ 2022 Rebollo and Tallon-Baudry

This is an open-access article distributed under the terms of the Creative Commons Attribution 4.0 International license, which permits unrestricted use, distribution and reproduction in any medium provided that the original work is properly attributed. 
1 The sensory and motor components of the cortical

2 hierarchy are coupled to the rhythm of the stomach

3 during rest

4 Abbreviated title: Anatomy of brain-stomach synchrony

5 Ignacio Rebollo ${ }^{1,2^{*}}$ and Catherine Tallon-Baudry ${ }^{1}$

6 1-Laboratoire de Neurosciences Cognitives et Computationnelles, Inserm, Ecole Normale

7 Supérieure, PSL University, 75005, Paris, France.

8 2-German Institute of Human Nutrition Potsdam-Rehbrücke, 14558, Nuthetal, Germany.

$9 \quad *$ Corresponding author: Ignacio Rebollo. Email: ignarebo@gmail.com

Number of pages: 40

Number of figures: 7

Number of tables: 1

Number of extended data tables: 2

Number of words: Abstract: 201; Introduction: 649; Discussion: 1795

Conflict of interest: The authors declare no competing interests. 


\section{Acknowledgements}

20 This work was supported by the European Research Council (ERC) under the European Union's

21 Horizon 2020 research and innovation program (grant agreement No 670325) to C.T.-B., as well as by

22 ANR-17-EURE-0017. I.R. was supported by a grant from DIM Cerveau et Pensée and Fondation

23 Bettencourt-Schueller. We thank Benoît Béranger and Juliette Klamm for their help during data 24 acquisition. 


\section{5 \\ Abstract}

Bodily rhythms appear as novel scaffolding mechanisms orchestrating the spatio-temporal organization of spontaneous brain activity. Here, we follow up on the discovery of the gastric restingstate network (Rebollo et al, 2018), composed of brain regions in which the fMRI signal is phasesynchronized to the slow $(0.05 \mathrm{~Hz})$ electrical rhythm of the stomach. Using a larger sample size $(\mathrm{n}=63$ human participants, both genders), we further characterize the anatomy and effect sizes of gastricbrain coupling across resting-state networks, a fine grained cortical parcellation, as well as along the main gradients of cortical organization. Most (67\%) of the gastric network is included in the somatomotor-auditory (38\%) and visual (29\%) resting state networks. Gastric brain coupling also occurs in the granular insula and, to a lesser extent, in the piriform cortex. Thus, all sensory and motor cortices corresponding to both exteroceptive and interoceptive modalities are coupled to the gastric rhythm during rest. Conversely, little gastric-brain coupling occurs in cognitive networks and transmodal regions. These results suggest not only that gastric rhythm and sensory-motor processes are likely to interact, but also that gastric-brain coupling might be a mechanism of sensory and motor integration that mostly bypasses cognition, complementing the classical hierarchical organization of the human brain.

Keywords: resting state networks, gastric, electrogastrogram, phase synchronization, cortical gradients, autonomic

\section{Significance statement}

While there is growing interest for brain-body communication in general and brain-viscera communication in particular, little is known about how the brain interacts with the gastric rhythm, the slow electrical rhythm continuously produced in the stomach. Here, we show in human 
participants at rest that the gastric network, composed of brain regions synchronized with delays to

49 the gastric rhythm, includes all motor and sensory (vision, audition, touch and interoception,

50 olfaction) regions, but only few of the transmodal regions associated with higher-level cognition.

51 Such results prompt for a reconsideration of the classical view of cortical organization, where the

52 different sensory modalities are considered as relatively independent modules. 


\section{Introduction}

55
Spontaneous brain activity is organized into networks of segregated regions displaying correlated activity across time (Biswal et al., 1995). While the study of resting state networks (RSNs) has proven fundamental to advance our understanding of the functional architecture of the brain (Power et al., 2014), there is still no consensus on RSN exact functions and computations. However, a distinction is often made between networks engaged in sensing and acting on the external world (Yeo et al., 2011), and more cognitive networks that have been associated with a wide spectrum of cognitive processes, ranging from episodic memory, prospective thinking and spontaneous cognition in the default network (Addis et al., 2007; Andrews-Hanna et al., 2014) to saliency detection, cognitive control, and attention in homonymous networks. The distinction between sensory and cognitive networks is not necessarily a sharp one and recently, the spatial layout of RSNs has been reframed in the context of gradients of macroscale cortical organization (Huntenburg et al., 2018) in which most of the spatial variance in cortical functional connectivity (Margulies et al., 2016), myelin (Huntenburg et al., 2017) and gene expression (Krienen et al., 2016) is explained by a connectivity gradient going from default network and transmodal regions to primary sensory-motor cortices.

The division of cortical regions into sensory-motor regions, required for the interaction with the external environment, and transmodal regions, devoted to higher-level cognition, is a recurrent feature not only in the resting-state network literature but also more generally in influential proposals of brain hierarchical organization (Felleman \& Van Essen, 1991; Mesulam, 1998; Markov et al., 2013). The organization of the brain in relatively independent sensory and motor modules coordinated by higher-order, transmodal regions leaves little space for the processing of internal bodily information. Despite mounting evidence that bodily rhythms and brain activity at rest are tightly coupled (Azzalini et al., 2019; Tort et al., 2018), RSNs are rarely related to internal, bodily information (for notable exceptions see (Chang et al., 2013; Shokri-Kojori et al., 2018). Recently, we reported the existence of coupling between brain activity at rest and the slow rhythm generated in 
the stomach (Rebollo et al., 2018; Richter et al., 2017), a finding which was replicated by an independent research group in a single participant scanned multiple times (Choe et al., 2020). The gastric rhythm is a slow $(0.05 \mathrm{~Hz})$ electrical oscillation that is intrinsically generated in the stomach wall by a specialized cell type, known as interstitial cells of Cajal (Sanders et al., 2014), and that can be measured non-invasively with cutaneous abdominal electrodes (Koch \& Robert M. Stern, 2004; Wolpert et al., 2020). The gastric rhythm is produced at all times but, during digestion, the amplitude of the rhythm increases through modulatory influences of the autonomous nervous system (Cruz et al., 2007; Sveshnikov et al., 2012; Travagli et al., 2006), setting the pace for the contraction of smooth muscles necessary to grind and mix ingested material and eject it into the small intestine.

In the present study, we present a follow-up on the gastric network, a novel resting state network composed of brain regions whose $\mathrm{fMRI}$ signals are phase synchronized to the gastric rhythm during resting fixation (Rebollo et al., 2018), in participants who were not in the active phase of digestion. We used a larger sample size $(n=63)$ to provide a detailed characterization of the anatomical extent of the human gastric network across the canonical seven resting state networks (Yeo et al., 2011) and across regions of a recent fine-graded multi-modal parcellation of the cerebral cortex (Glasser et al., 2016). We additionally computed effect sizes and compared them across regions, which could in turn guide sample size estimation for future studies.

We also quantified how the gastric network was positioned along the main two gradients of cortical organization (Margulies et al., 2016). Finally, we explored how different personal, physiological and experimental variables co-varied with the strength of gastric coupling across participants. 


\section{1 \\ Materials and Methods}

102

103

104

105

106

107

108

109

110

111

\section{Participants}

Seventy-two right-handed human participants took part in this study. Thirty-four participants took part in our first study (Rebollo et al., 2018, sample one) and an additional thirtyeight participants (sample two) were recruited for the present study. All volunteers were interviewed by a physician to ensure the following inclusion criteria: the absence of digestive, psychiatric or neurological disorders; BMI between 18 and 25, and compatibility with MRI recordings. Participants received a monetary reward and provided written informed consent for participation in the experiment and publication of group data. The study was approved by the ethics committee Comité de Protection des Personnes Ile de France III (approval identifier: 2007-A01125-48). All participants were instructed to fast for at least 90 min before the recordings. Data from nine participants were excluded. Three were excluded because excessive head movement during acquisition (translations larger than $3 \mathrm{~mm}$ or rotations larger than 3 degrees), three were excluded because their EGG spectrum did not show a clear peak that could allow us to identify the frequency of their gastric rhythm, and three more were excluded because less than $70 \%$ of their EGG cycles was within normogastric range (15-30 seconds per cycle, (Wolpert et al., 2020)). A total of 63 participants (mean age $23.95 \pm S D 2.76,31$ females and 32 males, and mean BMI $21 \pm S D$ 1.8) were included in the analysis described below.

\section{MRI data acquisition}

MRI was performed at 3 Tesla using a Siemens MAGNETOM Verio scanner (Siemens, Germany) with a 32-channel phased-array head coil. The resting-state scan lasted $900 \mathrm{~s}$ during which participants were instructed to lay still and fixate on a bull's eye on a gray background. Functional MRI time-series of 450 volumes were acquired with an echo-planar imaging (EPI) sequence and the following acquisition parameters: $T R=2000 \mathrm{~ms}, \mathrm{TE}=24 \mathrm{~ms}$, flip angle $=78^{\circ}, \mathrm{FOV}=204 \mathrm{~mm}$, and acquisition matrix $=68 \times 68 \times 40\left(\right.$ voxel size $\left.=3 \times 3 \times 3 \mathrm{~mm}^{3}\right)$. Each volume comprised 40 contiguous 
axial slices covering the entire brain. High-resolution T1-weighted structural MRI scans of the brain were acquired for anatomic reference after the functional sequence using a 3D gradient-echo sequence. The two samples had different anatomical sequences. The acquisition parameters for the anatomical scan of sample one were the following: TE $=1.99 \mathrm{~ms}, \mathrm{TR}=5000 \mathrm{~ms}, \mathrm{TI}-1=700 \mathrm{~ms} / \mathrm{TI}-$ 2=2500 ms, flip angle- $1=4^{\circ} /$ flip angle $-2=5^{\circ}, \quad$ bandwidth $=240 \mathrm{~Hz} /$ pixel, acquisition matrix $=240 \times 256 \times 224$, and isometric voxel size $=1.0 \mathrm{~mm}^{3}$, total anatomical sequence duration $=11$ minutes and 17 seconds. The acquisition parameters of sample two were the following: TE $=3.24 \mathrm{ms,}$ $\mathrm{TR}=2300 \mathrm{~ms}, \quad \mathrm{TI}-1=900 \mathrm{~ms} / \mathrm{flip} \quad$ angle- $1=9^{\circ}, \quad$ bandwidth $=210 \mathrm{~Hz} /$ pixel, $\quad$ acquisition matrix $=224 \times 256 \times 256$, and isometric voxel size $=1.0 \mathrm{~mm}^{3}$, with a total anatomical sequence duration of 5 minutes and 21 seconds.

\section{Physiological signal acquisition}

Physiological signals were simultaneously recorded during functional MRI acquisition using MRI compatible equipment and the same montage as in Rebollo et al (2018). Briefly, physiological signals were recorded $30 \mathrm{~s}$ before and after the resting state acquisition in order to avoid the spreading of the ringing artifact caused by the start of the MRI acquisition. The electrogastrogram (EGG) and electrocardiogram (ECG) were acquired using bipolar EMG electrodes (20 kOhms, $120 \mathrm{~cm}$ long) connected to a BrainAmp amplifier (Brain Products, Germany) placed between the legs of participants; the amplifier received a trigger signaling the beginning of each MRI volume. EGG was acquired at a sampling rate of $5000 \mathrm{~Hz}$ and a resolution of $0.5 \mu \mathrm{V} /$ bit with a low-pass filter of $1000 \mathrm{~Hz}$ and no high-pass filter (DC recordings). ECG was acquired at a sampling rate of $5000 \mathrm{~Hz}$ and a resolution of $10 \mu \mathrm{V} /$ bit with a low-pass filter of $1000 \mathrm{~Hz}$ and a high-pass filter of $0.016 \mathrm{~Hz}$. Prior to the recordings, the skin of participants was rubbed and cleaned with alcohol to remove dead skin, and electrolyte gel was applied to improve the signal-to-noise ratio. The EGG was recorded via four bipolar electrodes placed in three rows over the abdomen, with the negative derivation placed $4 \mathrm{~cm}$ to the left of the positive one. The midpoint between the xiphoid process and umbilicus was identified, and the first electrode pair was set $2 \mathrm{~cm}$ below this area, with the negative derivation set 
at the point below the rib cage closest to the left mid-clavicular line. The second electrode pair was set $2 \mathrm{~cm}$ above the umbilicus and aligned with the first electrode pair. The positive derivation of the third pair was set in the center of the square formed by electrode pairs one and two. The positive derivation of the fourth electrode pair was centered on the line traversing the xiphoid process and umbilicus at the same level as the third electrode. The ground electrode was placed on the left iliac crest. The ECG was acquired using three bipolar electrodes sharing the same negative derivation, set at the third intercostal space. The positive derivations were set at the fifth intercostal space and separated by $4 \mathrm{~cm}$.

\section{MRI preprocessing}

We used the same MRI preprocessing pipeline as described in Rebollo et al (2018). Brain imaging data were preprocessed using Matlab (Matlab 2017, MathWorks, Inc., United States) and the Statistical Parametric Mapping toolbox (SPM 8, Wellcome Department of Imaging Neuroscience, University College London, U.K.). Images of each participant were corrected for slice timing and motion with six movement parameters (three rotations and three translations). Each participant's structural image was normalized to Montreal Neurological Institute (MNI) template provided by SPM with affine registration followed by nonlinear transformation (Ashburner et al., 1999; Friston et al., 1995). The normalization parameters determined for the structural volume were then applied to the corresponding functional images. The functional volumes were spatially smoothed with a $3 \mathrm{~mm}^{3}$ fullwidth half-maximum (FWHM) Gaussian kernel.

The time series of voxels inside the brain, as determined using a SPM a priori mask, were subjected to the following preprocessing steps using the FieldTrip toolbox (Oostenveld et al., 2010) (Donders Institute for Brain, Cognition and Behaviour, Radboud University Nijmegen, the Netherlands. See http://www.ru.nl/neuroimaging/fieldtrip, release 06/11/2017). Linear and quadratic trends from each voxel time series were removed by fitting and regressing basis functions, and the BOLD time series were then bandpass filtered between 0.01 and $0.1 \mathrm{~Hz}$ using a fourth-order Butterworth infinite impulse response filter. A correction for cerebrospinal fluid motion was obtained 
by regressing out the time series of a $9 \mathrm{~mm}$ diameter sphere located in the fourth ventricle (MNI coordinates of the center of the sphere [0 -46 -32])

\section{EGG preprocessing}

Data analysis was performed using the FieldTrip toolbox. Data were low-pass filtered below $5 \mathrm{~Hz}$ to avoid aliasing and down-sampled from $5000 \mathrm{~Hz}$ to $10 \mathrm{~Hz}$. In order to identify the EGG peak frequency $(0.033-0.066 \mathrm{~Hz})$ of each participant, we first computed the spectral density estimate at each EGG channel over the $900 \mathrm{~s}$ of the EGG signal using Welch's method on $200 \mathrm{~s}$ time windows with 150 s overlap. Spectral peak identification was based on the following criteria: peaking power larger than $15 \mu \mathrm{V}^{2}$ and sharpness of the peak. Data from the selected EGG channel were then bandpass filtered to isolate the signal related to gastric basal rhythm (linear phase finite impulse response filter, FIR, designed with Matlab function FIR2, centered at EGG peaking frequency, filter width $\pm 0.015 \mathrm{~Hz}$, filter order of 5). Data were filtered in the forward and backward directions to avoid phase distortions and down-sampled to the sampling rate of the BOLD acquisition $(0.5 \mathrm{~Hz})$. Filtered data included $30 \mathrm{~s}$ before and after the beginning and end of MRI data acquisition to minimize ringing effects.

\section{Heart rate variability preprocessing}

In order to computer the power and ratio of Heart rate variability, we first removed the MRI gradient artefact from the ECG data using the FMRIB plug-in (lannetti et al., 2005; Niazy, Beckmann, lannetti, Brady, \& Smith, 2005, version 1.21) for EEGLAB (Delorme \& Makeig, 2004, version 14.1.1), provided by the University of Oxford Centre for Functional MRI of the Brain (FMRIB). Data from the ECG channels were then bandpass filtered $(1-100 \mathrm{~Hz})$ using a FIR filter, designed with Matlab function firws. We then retrieved the inter-beat-interval (IBI) time series by identifying $R$ peaks using a custom semi-automatic algorithm, which combined automatic template matching with manual selection of $\mathrm{R}$ peaks for extreme IBIs. Data from eleven participants was discarded at this stage due to noisy ECG recordings. The resulting IBI time series from the remaining 52 participants 
were then interpolated at $1 \mathrm{~Hz}$ using a spline function (order 3), and the average power in the low (0.06-0.15), and high (0.16-0.4) frequency bands was obtained by means of the Fourier transform using Welch's method on 120 s time windows with 100 s overlap.

\section{Experimental design and statistical analysis}

\section{Quantification of gastric-BOLD phase synchrony}

We used the procedure described in Rebollo et al (2018) in order to quantify Gastric-BOLD coupling. Briefly, the BOLD signals of all brain voxels were bandpass filtered with the same filter parameters as the ones used for the EGG preprocessing of each participant. The first and last 15 volumes ( $30 \mathrm{~s}$ ) were discarded from both the BOLD and EGG time series. The updated duration of the fMRI and EGG signals in which the rest of the analysis was performed was $840 \mathrm{~s}$. The Hilbert transform was applied to the BOLD and EGG time series to derive the instantaneous phases of the signals. The PLV (Lachaux et al., 1999) was computed as the absolute value of the time average difference in the angle between the phases of the EGG and each voxel across time (Equation 1)

$$
P L V_{x, y}=\left|\frac{1}{T} \sum_{t=1}^{T} e^{i(\varnothing x(t)-\varnothing y(t))}\right| \quad \text { Equation } 1
$$

Where $\mathrm{T}$ is the number of time samples, and $\mathrm{x}$ and $\mathrm{y}$ are brain and gastric time-series.

Statistical procedure for determining regions showing significant gastric-BOLD coupling at the group level

We employed a two-step statistical procedure adapted from previous work (Rebollo et al., 2018; Richter et al., 2017) in order to determine which voxels are significantly coupled to the stomach at the group level. We first estimated chance-level gastric-BOLD coupling at each voxel and in each participant. To estimate chance-level gastric-BOLD coupling, we computed gastric-BOLD coupling at each voxel between the BOLD data of the participant and the EGG data of the other 62 participants. For each participant and voxel, chance-level PLV was defined as the median of the 62 surrogate PLVs (Rebollo et al., 2018). Since only the empirical PLVs are specific to the frequency and 
phase of each participant gastric rhythm, our estimate of chance control for biases in PLV that could be expected using physiological signals with the same length, sampling rate, and frequency range as the empirical EGG, but which are not specific to the exact gastric frequency and phase of that participant. In the second step we used group-level statistics to determine regions in which gastricBOLD coupling was greater than chance. To compute group-level statistics, empirical and chancelevel PLVs were then compared using a cluster-based statistical procedure that intrinsically corrects for multiple comparisons (Maris and Oostenveld, 2007) implemented in FieldTrip. The procedure consists of applying t-tests between empirical PLV and chance-level PLV across participants at each voxel. Candidate clusters are formed by neighboring voxels exceeding a first-level t-threshold of $\mathrm{p}<0.005$ (one-sided). Each candidate cluster is characterized by the sum of the t-values in the voxels defining the cluster. To determine the sum of t-values that could be obtained by chance, we computed a cluster statistics distribution under the null hypothesis by randomly shuffling the labels 'empirical' and 'chance level' 1,000 times and applying the clustering procedure. At each permutation, we retained the largest positive and smallest negative summary statistics obtained by chance across all voxels and thus built a distribution of cluster statistics under the null hypothesis, and then assessed the empirical clusters for significance. Because the maximal values across the whole brain are retained to build the distribution under the null hypothesis, this method intrinsically corrects for multiple comparisons. Clusters with a Monte-Carlo $p<0.025$ (one-sided, corrected for multiple comparisons) were considered significant and are reported in the Results section.

\section{Quantification of gastric-bold shared variance}

To estimate the amount of variance in the BOLD signal that could be accounted for by gastric coupling, we computed, for each resting-state network, the squared coherence coefficient between the EGG and average BOLD time course across all voxels significantly coupled to the gastric rhythm using FieldTrip software. The coherence coefficient measures phase and amplitude consistency across time and is a frequency domain analog of the cross-correlation coefficient in the temporal 
domain. Therefore, its squared value can be interpreted as the amount of shared variance between two signals at a certain frequency (Bastos \& Schoffelen, 2016).

Anatomical characterization of the gastric network across resting state networks, cortical gradients, and individual regions

First, we calculated the effect sizes across significant gastric network voxels by computing Cohen's d (Equation 2) on difference between empirical and chance PLV, and then projected the resulting volume in $\mathrm{MNI}$ space to Freesurfer's average template (Desikan et al., 2006) using registration fusion (Wu et al., 2018).

Cohen's $d=\frac{t_{\text {empirical vs chance }}}{\sqrt{n}}$

Equation 2

where $t_{\text {empirical vs. chance }}$ is the t statistic of the paired t-test between empirical and chance level PLVs, and $n$ the number of participants.

We then used the seven RSN cortical parcellation available in freesurfer native space (Yeo et al., 2011) to identify the networks with more gastric network vertices and with the largest effect sizes. Variability in effect sizes was obtained via bootstrapping. We randomly picked 63 participants with replacement from our original sample, computed Cohen's $d$ and obtained the standard deviation of this metric across 1000 permutations.

In order to identify the individual regions comprising the gastric network, we used a recent multimodal parcellation of the cerebral cortex (Glasser et al., 2016). This parcellation consists of 180 areas per hemisphere and was obtained using a semi-automatized approach in multi-modal MRI data that detects sharp changes in cortical thickness, myelin, connectivity, and function. The parcellation was imported to freesurfer using the procedure and data available in: https://figshare.com/articles/HCP-MMP1_0_projected_on_fsaverage/3498446/2. For each region of the parcellation, we determined the overlap with the gastric network (percentage of the area in the gastric network), as well as the average effect sizes across significant gastric network vertices. 
We then compared the spatial layout of the gastric network with the first two gradients of functional connectivity described in (Margulies et al., 2016), which we retrieved from neurovault (https://identifiers.org/neurovault.collection:1598). We first resampled the volume containing gastric network significant voxels to match the voxel size of the gradients downloaded from Neurovault $\left(3 \mathrm{~mm}^{3}\right.$ to $\left.2 \mathrm{~mm}^{3}\right)$, using the tool imcalc from SPM. We then divided each gradient into one hundred equidistant bins and quantified for each bin the percentage of cortical voxels belonging to the gastric network. In order to test whether the gastric network is overrepresented in particular portions of each gradient, we created a distribution $(n=1000)$ of surrogate gastric networks located randomly across the cortex, and recomputed the overlap with each bin and gradient, obtaining a distribution the gastric network across the two gradients under the null hypothesis.

\section{Variability in gastric coupling across participants and regions}

We performed a series of exploratory analyses in order to test for associations between coupling strength, defined as the difference between empirical and chance level PLVs, and personal (gender, body-mass index, state anxiety score), physiological (EGG \& heart-rate variability characteristics) or experimental variables (time of day, elapsed time since last meal, head movement, a.k.a frame-wise displacement. Frame wise displacement was estimated as in (Power et al., 2012). State anxiety was measured using the STAI-B questionnaire (Spielberger et al., 1983). Gender and sample effects were assessed using unpaired samples t-test. The influence of the rest of the variables was assessed by separate robust linear regressions using R MASS package (Ripley et al., 2013), which included the covariate and the intercept term. Bayes factor was used to quantify the evidence for $\mathrm{HO}$ relative to $\mathrm{H} 1$, with a value $<0.33$ indicating substantial evidence for a null effect (Kass \& Raftery, 2012; Wetzels \& Wagenmakers, 2012). For the unpaired samples t-test we used the methods described in (Rouder \& Morey, 2011) to determine the Bayes factor. For the regression analysis, an approximation of the Bayes factor was computed (Wagenmakers, 2007) by comparing the Bayesian Information Criterion of the regression model including the covariate and the intercept to a 
regression model with only the intercept. Group level GLMs on coupling strength across voxels were performed using SPM 12 (Wellcome Department of Imaging Neuroscience, University College London, U.K.). All variables were mean centered and the intercept term was included.

Correction of $f M R I$ images for cardiac timing

As a control analysis, we computed the spatial extent of the gastric network and gastric coupling strength, with and without adjusting fMRI images for the cardiac cycle using retrospective image correction RETROICOR (Glover et al., 2000). We used the PhysIO toolbox implementation (Kasper et al., 2017) of the RETROICOR algorithm to generate six cardiac phase regressors from the cardiac timings in the 52 participants for whom we had cardiac peak timings. We then regressed out the cardiac regressors together with CSF timeseries, and obtained a new set of empirical and surrogate PLVs; as well as a new significant group level network (cluster forming threshold $p=0.005$ one-sided, cluster significance threshold $\mathrm{p}=0.025$, one-sided). We then compared the resulting gastric network with that of the same subsample but without RETROICOR correction, and calculated the distribution of the gastric network across Yeo 7 networks (Yeo et al., 2011). Finally, we compared how the RETROICOR pipeline affected coupling strength (empirical - surrogate PLV) in the original gastric network ( $n=63)$, for the 52 participants with and without RETROICOR.

\section{Code and overlays availability}

The code necessary to reproduce the results is available at https://github.com/irebollo/StomachBrain_2021. Unthresholded t-maps, effect sizes and significant voxels can be found at https://identifiers.org/neurovault.collection:9985 . 
335

Visualization tools

336

The distribution of coupling strength was plotted using raincloud plots (Allen et al., 2019).

337 Visualization of brain data on the cortical surface was done using Pysurfer 338 (https://pysurfer.github.io/). 
339

\section{Results}

\section{The stomach is synchronized with somatosensory, motor, visual and auditory regions}

We first determined the frequency of each participant gastric rhythm, by identifying the EGG channel displaying the largest peak within normogastric range $(0.033-0.066 \mathrm{~Hz})$. The mean EGG peak frequency across the sixty-three participants was $0.049 \mathrm{~Hz}( \pm \mathrm{SD} 0.004$, range $0.041-0.057)$. We did not find statistically significant differences between the two samples in EGG frequency (unpaired samples t-test, mean sample one $=0.047 \mathrm{~Hz} \pm \mathrm{SD} 0.0034 \mathrm{~Hz}$, mean sample two $=0.048 \mathrm{~Hz} \pm \mathrm{SD} 0.0036$ $\mathrm{Hz}, \mathrm{t}(61)=1.41, \mathrm{p}=0.161$ Bayes factor $=0.6$, indicating not enough evidence to disentangle between $\mathrm{H} 1$ and $\mathrm{H} 0$ ) nor in EGG power (unpaired samples t-test, mean sample one $=220 \mu v^{2} \pm \mathrm{SD} 410 \mu v^{2}$, mean sample two $=333 \mu v^{2} \pm S D 530^{2} \mu v, t(61)=1.41, p=0.356$, Bayes factor $=0.37$, indicating not enough evidence to disentangle between $\mathrm{H} 1$ and $\mathrm{HO}$ ). We did find differences in the standard deviation of EGG cycle length, which was significantly smaller in sample two than sample one (unpaired samples $t$-test, mean sample one $=3.38$ seconds \pm SD 1.25 seconds, mean sample two $=2.66$ seconds $\pm S D 1.48$ seconds, $t(61)=-2.08, p=0.041)$, indicating a more stable gastric rhythm in sample two.

We then quantified the degree of phase synchrony between the EGG signal and BOLD time series filtered around gastric frequency using empirical and chance-level estimates of phase-locking value (PLV). PLV (Lachaux et al., 1999) measures the level of phase synchrony, defined as the stability over time of the time lag between two time series, independently of the amplitude of the two timeseries. Importantly, PLV is large as long as the time lag is constant, independently from the value of the time lag, and does not provide information about directionality. In each participant and voxel, we computed the empirical PLV between the narrow-band BOLD signals and the gastric rhythm. We also estimated the amount of PLV that could be expected by chance in each voxel using the BOLD signal of one participant and the EGG of the other sixty-two participants, and taking the median of that surrogate distribution as chance-level PLV. The empirical PLVs were then compared with the chance- 
level PLVs using a cluster-based statistical procedure that intrinsically corrects for multiple comparisons (Maris \& Oostenveld, 2007). Significant phase coupling between the EGG and restingstate BOLD time series occurred in thirty-two clusters (voxel threshold $p<0.005$, one-sided paired ttest between observed and chance PLV; cluster threshold corrected for multiple comparisons, Monte-Carlo $p<0.025$ one-sided). Exact $p$-values are reported for each cluster in supporting table Figure 1-1.

As observed before (Rebollo et al., 2018) the gastric network (Figure 1A, Figure 1-1), comprises bilateral regions along the central, cingulate and lateral sulci, as well as occipito-parietaltemporal regions and portions of the left striatum (Figure 1B), bilateral thalamus (Fig. 1C) and cerebellum (Fig. 1D). The distribution of the average gastric network coupling strength (defined as the difference between empirical and chance PLV) across participants (Fig 1F) ranged from 0.0026 to 0.2096 (mean=0.0417, STD=0.0399, median =0.0319). We found no difference between the two samples in average coupling strength (unpaired sample t-test, mean sample one $=0.043 \pm S D 0.044$, mean sample two $=0.039 \pm S D 0.031, t(61)=-0.42, p=0.670, B F=0.27$, indicating substantial evidence for $\mathrm{HO})$.

We then used a well-known parcellation of the cortical surface into seven resting state networks (Yeo et al., 2011) in order to quantify the overlap of the gastric network with canonical resting state networks. This analysis (Fig 1E) confirmed that most of the gastric network $(67 \%)$ is included in the somato-motor (38\%) network, which also includes the auditory cortices, and in the visual (29\%) network. Gastric coupling in the somato-motor-auditory network spans somatosensory, motor and auditory regions surrounding the central, lateral and cingulate sulci. Gastric coupling in the visual network spans striate and extrastriate visual regions, and is particularly pronounced in medial and ventral occipital regions. Effect sizes (Cohen's d of paired t-test of empirical against chance PLV) were similar across visual and somato-motor networks (Fig. 1E, right). Effects sizes in the few coupled regions of the default, saliency, control and attention networks were only slightly 
smaller than those in sensory and motor cortices (Fig. 1E, right). Similarly, the average shared variance, as estimated from squared coherence, between the EGG and BOLD signal in each RSN, was very similar in all RSNs, ranging from $13.8 \pm 2.0 \%$ in the limbic system to $15.8 \pm 2.2 \%$ in the default network. Intersubject variability was high in all RSNs, with shared variance in individual participants ranging from below $1 \%$ up to above $40 \%$ in all RSNs.

The gastric network showed a very limited overlap with non-sensory networks (Fig. 1E, left). The overlap with the default network (9.5\%) occurs mostly in one medial node of the default network, the retrosplenial cortex as well as in the lateral node in the superior temporal sulcus, and a small rostral prefrontal region. Only $8.1 \%$ of the gastric network is found in the saliency network, sparing core regions of the saliency network such as the anterior insula and the fundus of the dorsal anterior cingulate sulcus. Only very few regions of the gastric network belonged to the control network (4.6\%), dorsal attention network (3.6\%) or limbic network $(0.4 \%)$. Subcortical regions, (which in this parcellation include thalamus and striatum but not cerebellum), represented $6.8 \%$ of the gastric network. Even if the percentage of the gastric network in subcortical regions is small, effect sizes were similar as those in the cortical regions of the gastric network (Fig $1 \mathrm{E}$ ). We do not examine thalamus and striatum in more details because the voxel size we used is not well suited for those structures, and the projection of voxels into surfaces is optimized for cortical regions and hence induce deformation on subcortical regions. Finally, $11.9 \%$ of the gastric network is found in the cerebellum. Note however that the most ventral part of the cerebellum was not imaged in this study, so this percentage is only an indication of the share of the cerebellum in the gastric network. Within the cerebellum, the gastric network was mostly found in Left crus I (30.1\%), Right lobule VI (16.2\%), Left crus II (11.9\%), Left lobule VI (11.57\%), Vernus crus II (10.4\%), according to the parcellation from (Diedrichsen et al 2009).

While the gastric network is spatially extended, effect sizes remain moderate. The average Cohen's d across visual, somatomotor or default network voxels was close to 0.4 , raising the issue of 
replicability and required sample sizes. For the first-level statistical threshold employed in these analysis (cluster forming threshold $=0.005$ one-sided), a sample size of at least 77 participants is required to observe an effect of this magnitude $80 \%$ of the time, meaning that it is possible that the current study is slightly underpowered. Still, we wanted to verify whether gastric coupling was more prevalent in sensory-motor cortices across the two subsamples that encompass this study. For this, we recomputed the spatial layout and effect sizes of significant gastric brain coupling in each of the two subsamples (29 and 34 participants respectively), albeit using a more liberal statistical threshold (cluster forming threshold $=0.05$ one-sided), which requires a sample size of 41 participants to detect an effect $80 \%$ of the time. The spatial layout of the gastric coupling was indeed similar across the two sub-samples (Fig. 1E), with the largest proportion of the gastric network in somato-motor and then visual networks, and more varying proportion of coupling in transmodal networks across the two subsamples. The relative strength of effect sizes was more variable between the two sub samples, with sample 1 having larger effect sizes in visual than somato-motor networks and an opposite pattern in sample 2.

To verify that the dominance of sensory and motor cortices in the gastric network was not related to the level of details of the parcellation, we used a more recent parcellation of the cerebral cortex (Glasser et al., 2016) consisting of 180 areas per hemisphere. The parcellation was obtained using a semi-automatized approach in multi-modal MRI data that detects sharp changes in cortical thickness, myelin, connectivity, and function. For each region of the parcellation overlapping with the gastric network, we computed the percentage of the area in the gastric network and the average effect sizes across significant gastric network vertices (Extended data in figure 1-2). The 25 regions with the largest effect sizes across both hemispheres (Figure $1 \mathrm{G}$ ), were primary motor and somatosensory cortices (Regions 4, 3b, 1 and 3a), premotor (6d, SCEF, 6mp, FEF), cingulate motor (24dd), auditory (STSda, A5, MBelt, A1, PBelt, RI, STGa), insular granular, opercular (area 43) early visual regions (V1, V2, V3 and VVC), area FEF from the saliency/attentional networks, and the 
retrosplenial complex (RSC), area STSda and area 10d, three regions typically associated with the default network.

Because the whole brain analysis indicates that all sensory cortices are coupled to the gastric network except the olfactory cortex, we performed a targeted region of interest analysis of the left and right piriform cortex as defined in the (Glasser et al., 2016) parcellation. We found that coupling in the olfactory cortices has indeed a medium effect size (Cohen's $d$ left $=0.56$, right $=0.61$ ), comparable to the effect sizes in primary visual (left $=0.57$, right $=0.54$ ) or auditory (left $=0.67$; right $=$ $0.61)$ cortices. The absence of olfactory cortices from the whole brain analysis is thus probably due to the small size of those cortices.

\section{Gastric network and cortical gradients of functional connectivity}

The gastric network is mostly found in somato-motor-auditory and visual RSNs. To verify that this is not due to the specific resting-state network parcellations we used, or to a somewhat arbitrary division between sensory and transmodal areas, we analyzed how the gastric network is distributed along the first two gradients of functional connectivity described by (Margulies et al., 2016). In this approach, each cortical voxel can be defined by its location along two different axes, one that goes from unimodal to transmodal regions, and another one that goes from visual to transmodal to somato-motor-auditory regions. In Figure 2A, we reproduce the findings of (Margulies et al., 2016). When considering the whole cortex, the projection of the probability density on the first gradient shows two prominent peaks at the extremities, corresponding to transmodal and unimodal regions (Figure 2A, red curve). When considering only the gastric network, the probability density shows a markedly different profile, with an increase in the unimodal extreme only (Figure 2B, red curve). As described by Margulies et al (2016), the projection of the whole brain probability density on the second gradient shows a prominent peak in transmodal regions (Fig 2A blue curve). However, gastric network voxels are more densely represented in the visual and somato-motor-auditory 
extremes of the gradient (Fig 2B blue curve), indicating that coupling with the gastric rhythm is more likely to be present in unimodal than in transmodal brain regions.

We then tested whether the distribution of gastric network voxels along the first and second gradients could be due to chance. We first computed the percentage of brain voxels that belonging to the gastric network for each of the two gradients across one hundred equidistant bins

(Fig 2C, orange lines). In order to test whether spatial biases of such size could be obtained by chance, the spatial position of the gastric network voxels was randomly permuted across the cortex, while keeping the spatial layout of the gradients intact, and computed the percentage of voxels belonging to the surrogate gastric network thus created. The procedure was repeated one thousand times to estimate the distribution under the null hypothesis (Fig 2C, black circles). For the first gradient, the percentage of all brain voxels overlapping with the gastric network across bins is systematically larger than chance in the unimodal bins of the gradient, and systematically smaller in the remaining bins (Fig $2 \mathrm{C}$ left). Similarly, for the second gradient, the overlap with the gastric network across bins is systematically larger than chance in the unimodal bins and systematically smaller in the transmodal bins (Fig $2 \mathrm{C}$ right). This analysis confirmed that the spatial biases in the unimodal extremes of the gradients could not be obtained by chance.

\section{Anatomical characterization and effect sizes in the gastric network}

We now examine in more detail the anatomy of the gastric network, starting with regions belonging to the somato-motor network in central and mid-cingulate regions, followed by a characterization of opercular regions in and around the lateral sulci, including auditory cortices and insula, then regions located in the posterior portion of the medial wall and in occipital, posterior cingulate, temporal and parietal cortices, and ending with the few transmodal regions in frontal, prefrontal and lateral temporal cortices.

Gastric network surrounding the central and cingulate sulci 
Gastric network in central and mid-cingulate regions in central and mid-cingulate regions (Figure 3A), the gastric network covers primary somatosensory (green), motor, premotor, and cingulate motor (blue) and non-motor (violet) cingulate regions, as well as area 55b (pink). The primary somatosensory cortex proper (areas $3 \mathrm{~b}$ and 1 ), is located in the postcentral gyrus and is involved not only in the representation of bodily surface but also of the viscera (Amassian, 1951; Azzalini et al., 2019; Dum et al., 2009; M. Kern et al., 2013). Accessory somatosensory area 2, in the bank of the post-central sulcus, shows little coupling with the gastric rhythm. In the anterior direction, area 3a, in the fundus of the central sulcus, is sometimes referred to as part of the somatosensory cortex but can also be seen as a transition zone with primary motor area 4 (Catani, 2017) in the posterior bank of the postcentral gyrus. Besides its role in controlling skeletal muscle, area 4 has been shown to provide sympathetic input to the stomach (Levinthal \& Strick, 2020). Both area $3 a$ and area 4 display coupling with the gastric rhythm. The gastric network is also found in numerous premotor areas, generally involved in integrating sensory and motor information for the performance of actions: area $6(6 \mathrm{~m}, 6 \mathrm{~d}, 6 \mathrm{v}, 6 \mathrm{a})$, the frontal eye field (FEF) and supplementary motor areas (6mp, 6ma, SCEF). Note that area SCEF (supplementary motor and cingulate eye fields), extends into the dorsal bank of the anterior cingulate sulcus. Along the medial wall, the gastric network overlaps with left area 5 I (cyan), the cingulate motor areas (24dd, $24 d v$, blue), known to contain several somatotopic maps (Amiez \& Petrides, 2014) and to receive spinothalamic input (Dum et al., 2009), the paralimbic cortex (area p32pr), areas p24pr and 23d, located adjacent to the corpus callosum and area 23c, located between the cingulate motor regions and the task-negative precuneus Finally, the gastric network also includes area 55b mostly on the right side, a recently discovered region which is left-lateralized for language and right-lateralized for theory of mind (Glasser et al., 2016), and to a lesser extent, the superior frontal language area (SFL), a region that is also left-lateralized for language.

Regions containing a large proportion of voxels belonging to the gastric network (Figure 3B) include primary somatosensory (3b, 3a, 1), primary motor (4), premotor (SCEF, 6d, FEF), cingulate 
motor regions (24dd), region $55 \mathrm{~b}$ and area $5 \mathrm{~m}$. Gastric-brain coupling show a strong right lateralization in regions surrounding the cingulate sulcus (p32pr, p24pr, 23cc 23d, 24dv, 24dd, SCEF), as well as in FEF and area 55b, while areas 1 and $5 \mathrm{~m}$ are left-lateralized. Effect sizes (Figure 3C) were largest in somatosensory (3b, 1, 3a) and primary motor (4) cortices, followed by premotor and cingulate motor regions (6d, SCEF, 24dd, FEF, 6m). Asymmetries in effect sizes showed similarities with asymmetries in overlap, with right lateralization in effect size in cingulate regions (p24pr, 23cc 23d, 24dv, 24dd, SCEF) and region 55b, and left lateralization in regions 5I, 5m and 2.

\section{Gastric network surrounding the lateral sulcus}

In and around the lateral sulcus (figure 4A), the gastric network spans secondary somatosensory (green), frontal operculum (blue), early (dark violet) and association (light violet) auditory regions as well as regions of the insula proper (pink). The secondary somatosensory (SII), known to process inputs from the skin but also from the viscera (Amassian, 1951) is composed of areas OP1, OP2-3, and OP4, and is located anteriorly to area Pfcm, in the inferior parietal cortex. SII is separated from the frontal operculum (areas FOP1, FOP2, FOP3, and FOP4) by area 43 (cyan). Early auditory regions, involved in the processing, identification and location of sounds (Jasmin et al., 2019), include the primary auditory cortex (A1) and the surrounding lateral belt (LBelt), medial belt (MBelt), parabelt (PBelt) and retro-insular cortex (RI). Auditory association regions extend inferiorly to the superior temporal sulcus and include A4, A5, Temporal Area 2 (TA2), and auditory default network regions dorsal posterior superior temporal sulcus (STSdp), dorsal anterior superior temporal sulcus (STSda), and ventral anterior superior temporal sulcus (STSva). The insula proper consists of area 52, a transition region between auditory cortex and insula, the granular insula (Ig), which contains a complete somatotopic motor map (Glasser et al., 2016) as well as viscerosensitive neurons (Cechetto \& Saper, 1987), the middle insular area (MI), and posterior insular area I and II (Pol1, Pol2). 
The regions with the largest overlap with the gastric network consist of early auditory regions (Pbelt, A1, MBelt, RI), right insular regions (Ig, 52), left parietal opercular regions (OP4, OP1, 43) and right area FOP1 (Figure 4B). Effect sizes were largest in early and association auditory regions (A5, A4, MBelt, LBelt, A1, PBelt, STSda, RI, TA2), insular cortex (52, Ig, Pol2) and areas 43 and FOP1 (Figure 4C). Overall, overlap and effect sizes in SII (OP1, OP2-3) were most prominent in the left hemisphere while overlap and effect sizes in the frontal, insular, MBelt and auditory association regions were strongly right-lateralized.

\section{Gastric network in posterior regions}

In the posterior part of the brain, the gastric network covers large portions of the occipital visual cortex, (Figure 5A, dark green), occipito-parietal sulcus (blue) and retrosplenial cortex (violet), dorsal precuneus (light green), ventral visual stream (pink), and right temporo-parietal-occipital junction (black). The gastric network is found in all early visual regions (V1, V2, V3, and V4), involved in the processing of low-level visual properties such as orientation and color, extending superiorly to the dorsal stream (V3A, V3B, V6, V6A, V7), involved in the location of objects in space, up to area 7Am and the precuneus visual area (PCV), a region sensitive to optic flow (Glasser et al., 2016). Medially and anteriorly, the gastric network extends to the parieto-occipital sulcus and recruits portions of the dorsal visual transition area (DVT), prostriate cortex (Pros), and parieto-occipital sulcus regions 1 and 2 (POS1, POS2), regions typically involved in the generation of the electrophysiological alpha rhythm (Richter et al., 2017) and in vigilance fluctuations. Area POS2 stands out as due to its large overlap with the gastric network (90\%). Conversely, it is worth noting that the gastric network spares most of the ventral precuneus (Yellow; areas 7m, 31pd, 31a, 31pv), which is one of the core nodes of the default network. Ventrally, the gastric network extends to ventromedial visual areas 1, 2 and 3 (VMV1, VMV2, and VMV3), as well as regions of the ventral stream, involved in the processing of objects identity (V8, area lateral occipital 2, LO2, posterior inferotemporal complex, PIT, and fusiform face complex, FFC), and the occipital-temporal-parietal junction (Black, MT, Pgp, TOPJ3, LO3) in the right hemisphere. 
A cluster of dorsal right regions (V3A, V6A and V7, POS2 and DVT) stands out, with more than $80 \%$ of those regions in the gastric network (Figure 5B). Other posterior regions with large overlap were right area VMV3, left V3B, and, to a lesser extent, the RSC, as well as left V1 and ProS. Effect sizes (Figure 5C) were largest in RSC, right FFC and most occipital visual regions including V1. A rightwards lateralization in effect sizes and overlap was present for most regions, with the exception of area V3B, which displayed a leftwards lateralization.

\section{Gastric network in prefrontal and lateral temporal cortex}

The gastric network includes a few transmodal regions located in the middle temporal gyrus (Figure 6A, pink), inferior frontal cortex (blue), dorsolateral prefrontal cortex (green), and in the orbitofrontal cortex (yellow). In the middle temporal gyrus, the gastric network is found in the middle temporal gyrus (TE1m, TE1p), as well as in ventral regions of the superior temporal sulcus (STSdp, STSvp), belonging to the auditory portion of the default network. The percentage of voxels belonging to those regions remain small (Figure 6B), with moderate effect sizes (Figure 6C), and a marked right lateralization

In right inferior frontal cortex (Figure 6A, blue), the gastric network forms a cluster that comprises the posterior portion of the inferior frontal sulcus (IFSp), the anterior portion of the inferior frontal junction (IFJa), and area posterior 9-46d (p9-46d) as well as area 44, all regions of the control network, extending to rostral area $6(6 r)$ of the saliency network. In the left dorsolateral prefrontal cortex (Figure 6A green), the gastric network includes multiple regions of the control network, including Superior Transitional Area 6-8 anterior (s6-8a), anterior area 10p (a10p), area 11 lateral (11l), anterior area 9-46 ventral (a9-46v), area 9-46 dorsal (9-46d), as well as area 8b lateral from the default network and polar area 10p (p10p) from the limbic network. Overlap is particularly pronounced in a10p and a9-46v (Figure 6B) with an exclusively left lateralization. Finally, in orbitofrontal cortex (Figure 6A yellow), the gastric network includes dorsal and rostral portions of area $10(10 d, 10 r)$ from the default. 
591

\section{Variability in gastric coupling across participants and regions}

We examined how differences in personal (gender, body-mass index, state anxiety score), physiological (EGG \& heart-rate variability characteristics) or experimental variables (time of day, elapsed time since last meal, head movement, a.k.a frame-wise displacement), were related to variability in coupling strength across participants, in a series of exploratory analyses. Due to the small age range of our sample (18-30), we did not include age as a regressor. We first tested whether any of these variables accounted for a compact measure, the mean coupling strength in the gastric network (Table 1). We found no differences between genders in the average coupling strength (mean female $=0.039 \pm S D$ 0.036, mean male $=0.044 \pm S D 0.044$, paired $t$-test, $t(61)=0.48, p=0.634$, Bayes factor $=0.279$, indicating substantial evidence for the null hypothesis). All other variables were tested with robust linear regressions. With one exception, none of the variables tested accounted for coupling strength, including state anxiety $(t(58)=0.47, p=0.634$, Bayes factor $=0.124$ indicating substantial evidence for the null hypothesis).

The only variable related to coupling strength was the low frequency component of heartrate variability (LF-HRV, Figure 7C), which was associated with a larger brain coupling $(n=52, t(50)=$ 3.76 , robust regression, $r^{2}=0.22$, uncorrected $p=0.0004$, Bonferroni corrected $p=0.0048$ ). LF-HRV reflects multiple cardiac control mechanisms, including blood pressure and baroreflex activity, and is under the control of both sympathetic and parasympathetic nervous system (Goldstein et al., 2011; Reyes del Paso et al., 2013). Because coupling strength turns out to be related to heart rate variability, we recomputed to the spatial layout of the gastric network with and without correction for cardiac pulsation using RETROICOR (Glover et al., 2000; Kasper et al., 2017), in the 52 participants where heart rate time series were obtained. We observed no overall difference in the distribution of the gastric network across resting networks: gastric-brain coupling was mostly observed in somatomotor-auditory network as well as in the visual network after RETROICOR correction (Figure 7D). The 
615

spatial layout of the gastric network is thus not solely driven by cardiac pulsation effects. We then tested whether correcting for cardiac pulsation effects affected the overlall level of coupling strength. We compared coupling strength in the same spatial extent of gastric network (as defined on 63 participants), with and without RETROICOR. Correcting for cardiac pulsation induced a significant reduction in overall coupling strength (Figure 7E, mean before RETROICOR $=0.043 \pm$ STD 0.040, mean after RETROICOR $=0.026 \pm$ STD 0.020, paired t-test $t(51)=5.42, p=0.000001)$. The linear relationship between LF-HRV and coupling strength remained significant after cardiac pulsation correction $\left(n=52, t(50)=4.23\right.$, robust regression, $\left.r^{2}=0.26, p=0.00009\right)$.

We reasoned that only some gastric network regions might be modulated by the variables we analyzed. We therefore used two different strategies to look for more subtle effects of interindividual variables on coupling strength, both accounting for multiple comparisons. We first tested for effects of these variables across Glasser et al (2016) ROls overlapping with the gastric network, applying false-discovery rate correction over the 226 ROls tested for each variable separately. We only found one significant association, with coupling strength in right area 7Am co-varying with EGG cycle duration variability $(t(61)=3.936, F D R p=0.048)$. We also performed group level general linear models with coupling strength in all brain voxels as the dependent variable. Gender, group, BMI, frame-wise displacement, time of the day, EGG frequency, EGG power were used in a first general linear model with all participants $(n=63)$. Separate general linear models including these variables and either state anxiety $(n=61)$, elapsed time since last meal $(n=51)$ or heart rate variability measures $(n=51)$ were performed. None of the models survived correction for multiple comparisons (FWE $p<0.05)$

\begin{tabular}{|c|c|c|c|c|c|c|c|c|c|c|}
\hline $\begin{array}{c}\text { Variable } \\
\text { type }\end{array}$ & $\begin{array}{c}\text { Variable } \\
\text { name }\end{array}$ & $\mathrm{n}$ & $\min$ & $\max$ & $\operatorname{mean}$ & median & Std & $\mathrm{t}$ & $\mathrm{p}$ & $\mathrm{BF}$ \\
\hline \multirow{2}{*}{ Personal } & \begin{tabular}{c} 
Gender \\
\cline { 2 - 10 }
\end{tabular} & $\begin{array}{c}\mathrm{f}=32, \\
\mathrm{~m}=31\end{array}$ & & & & & & 0.48 & 0.634 & 0.2799 \\
\cline { 2 - 10 } & $\begin{array}{c}\text { Sody mass } \\
\text { index } \\
\text { (STAI B) }\end{array}$ & 63 & 17.5 & 24.5 & 21.0 & 21.0 & 1.8 & 0.52 & 0.604 & 0.0000 \\
\hline
\end{tabular}




\begin{tabular}{|c|c|c|c|c|c|c|c|c|c|c|}
\hline \multirow{3}{*}{ Experimental } & $\begin{array}{l}\text { Time of day } \\
\text { (minutes } \\
\text { since } \\
\text { midnight) }\end{array}$ & 63 & 581 & 1100 & 812 & 771 & 150 & 0.10 & 0.919 & 0.1325 \\
\hline & $\begin{array}{l}\text { Time since } \\
\text { last meal } \\
\text { (hours) }\end{array}$ & 52 & 1 & 8 & 4.0 & 3.5 & 2.0 & 1.24 & 0.220 & 0.0853 \\
\hline & $\begin{array}{c}\text { Frame-wise } \\
\text { displacement } \\
(\mathrm{mm})\end{array}$ & 63 & 0.061 & 0.323 & 0.123 & 0.111 & 0.052 & 0.64 & 0.527 & 0.2635 \\
\hline \multirow{6}{*}{ Physiological } & $\begin{array}{c}\text { EGG } \\
\text { frequency } \\
(\mathrm{Hz})\end{array}$ & 63 & 0.041 & 0.057 & 0.048 & 0.049 & 0.004 & 0.60 & 0.550 & 0.0000 \\
\hline & $\begin{array}{c}\text { EGG power } \\
\left(\mu v^{2}\right)\end{array}$ & 63 & 6 & 3056 & 281 & 107 & 479 & 0.18 & 0.858 & 0.0000 \\
\hline & $\begin{array}{c}\text { EGG cycle } \\
\text { duration } \\
\text { variability (s) }\end{array}$ & 63 & 1 & 6.5 & 3 & 2.8 & 1.4 & 0.93 & 0.357 & 0.0605 \\
\hline & $\begin{array}{c}\text { LF-HRV } \\
\text { power }\left(\mathrm{ms}^{2}\right)\end{array}$ & 52 & $0.5 *-10^{5}$ & $4.5 *-10^{5}$ & $9.5 *-10^{5}$ & $0.7 *-10^{5}$ & $0.7 *-10^{5}$ & 3.76 & 0.0004 & 3099 \\
\hline & $\begin{array}{c}\text { HF-HRV } \\
\text { power }\left(\mathrm{ms}^{2}\right)\end{array}$ & 52 & $0.4 *-10^{5}$ & $3.3 *-10^{5}$ & $5.5 *-10^{5}$ & $0.3 *-10^{5}$ & $0.5 *-10^{5}$ & 0.04 & 0.962 & 0.1463 \\
\hline & $\begin{array}{l}\text { LF/HF HRV } \\
\text { ratio }\end{array}$ & 52 & 0.339 & 8.15 & 2.51 & 1.91 & 1.97 & 1.87 & 0.067 & 0.3234 \\
\hline
\end{tabular}

636

Table 1: Variables tested to account for average coupling strength in the gastric network. For each

variable tested the table displays the descriptive statistics, $t$-values, uncorrected $p$ values, and Bayes

factors (BF), for which values smaller than 0.33 indicates substantial evidence for the null hypothesis.

Gender effects were assessed by comparing males and females with an unpaired sample t-test, all

other variables were assessed using robust linear regressions. Bold font indicates significant

differences from the null distribution for $\mathrm{p}$ values and substantial evidence for the null hypothesis for 


\section{Discussion}

We examined the anatomy of the gastric network, a set of brain regions phase coupled to the rhythm of the stomach during rest, and analyzed the spatial layout of the gastric network at the level of its constituent regions (Glasser et al., 2016), resting-state networks (Yeo et al., 2011), and its extent and position along the first two gradients of cortical connectivity that underlie the topological structure of the cortex (Margulies et al., 2016). We found that the gastric network is overrepresented in unimodal sensory-motor regions, and underrepresented in transmodal regions. All sensory and motor cortices are coupled to the gastric rhythm, including not only areas responding to touch, vision and audition but also the interoceptive insula and, to a lesser extent, the olfactory piriform cortex. Only few transmodal regions were coupled to the gastric rhythm, mostly in the default network (retrosplenial cortex, right area STSda). We also observed significant coupling in the cerebellum. None of the personal, physiological and experimental variables tested co-varied with the overall strength of gastric coupling across participants, with the exception of an index of cardiac autonomic activity. Notably, we found substantial evidence for an absence of association between state anxiety and gastric-brain coupling during rest.

\section{Anatomical pathways for gastric brain coupling}

Some of the gastric network regions we observe are known to be involved in interoception and autonomic functions. Such is the case of the granular insula, the somatosensory cortices, and the cingulate motor regions (Amassian, 1951; Cechetto \& Saper, 1987; Dum et al., 2009), which receive visceral inputs, and motor regions, which provide sympathetic input to the stomach (Levinthal \& Strick, 2020). However, we find that gastric-brain coupling extends well beyond expected visceral processing regions, notably early and association visual and auditory regions. Such results are in line with findings in rats, where the electrical stimulation of the stomach elicits BOLD responses not only 
670

671

672

673

674

675

676

677

678

in somatosensory, insula, cingulate cortices, but also in motor, auditory and visual cortices (Cao et al., 2019). Changes in visual cortices activity has also been observed in humans in response to painful gastric distension (Van Oudenhove et al., 2009), subliminal rectal distension (M. K. Kern \& Shaker, 2002), as well in dogs after gastric electrical stimulation (Yu et al., 2014). Similarly, colonic pain induces a response in the rat auditory cortex (Z. Wang et al., 2008). The parabrachial nuclei, the major relay of both spinal and vagal visceral afferents, projects not only to thalamic relay nuclei involved in interoception and touch (Coen et al., 2012; Saper \& Loewy, 1980), but also to the visual lateral geniculate nucleus (Erişir et al., 1997), and potentially to the auditory medial geniculate nuclei (Uhlrich et al., 1988). Projections of the parabrachial nucleus might thus at least partially mediate gastric-BOLD coupling in sensory cortices. It is also worth underlining that we find no gastric-BOLD coupling in anterior insular and medial prefrontal regions, two regions involved in parasympathetic control of the stomach in rats (Levinthal \& Strick, 2020).

Beyond direct ascending or descending communication with the stomach, gastric-coupling could also stem from intra-cortical connectivity. For instance, direct connections have been reported between secondary somatosensory cortex to auditory regions, between visual area MT and primary somatosensory (Cappe \& Barone, 2005). Whether and how our findings pertain to interoceptive prediction models (Barrett \& Simmons, 2015; Petzschner et al., 2021) remains an open question, since neither the spatial resolution nor the gradients (Huntenburg et al., 2018; Margulies et al., 2016) we used provide information on the laminar origin of gastric-BOLD coupling. Finally, neuromodulation might also be involved (Rebollo et al., 2021; Rinaman \& Schwartz, 2004). In particular, activity in the locus coeruleus, the main source of norepinephrine to the forebrain, is modulated by gastric, colonic and bladder distension (Elam et al., 1986; Saito et al., 2002), inducing fluctuations in arousal that can also be obtained with distension of the small intestine (Kukorelli \& Juhász, 1977). 


\section{Intersubject variability and cardiac activity}

When examining effect sizes and the required sample sizes needed to observe our effect, it appears that the current study might still be slightly underpowered. However, note that we could only estimate the sample size for the first step of our statistical procedure (voxel-level statistics), and not to the second step (cluster-level statistics). A more precise estimation of the required number of participants for future studies remains to be established, considering the intersubject variability observed in gastric-BOLD coupling that was not readily explained by the individual variables we explored.

Indeed, we examined a number of individual variables which could covary with gastricbrain coupling, including state anxiety, which has often been associated with (mostly cardiac) interoception (Domschke et al., 2010). We found either robust evidence for no covariation using Bayesian statistics, or no evidence for covariation, despite having a sample size large enough to detect moderate effect sizes. Our experimental setting was designed to minimize variation in digestive and autonomic state. Therefore, we cannot rule out the possibility that BMI, hunger or state anxiety influence gastric-brain coupling in participants with larger BMIs or state anxiety levels, or if we had explicitly manipulated state anxiety or hunger in a within subject-design.

The only variable displaying a significant association with gastric-brain coupling was the LF component of HRV. LF-HRV reflects multiple cardiac control mechanisms, including variations in blood pressure and baroreflex activity (Goldstein et al., 2011; Reyes del Paso et al., 2013). LF-HRV is a known modulator of spontaneous fMRI activity, even after correction for cardiac pulsation effects (Shmueli et al., 2007), in line with the preserved correlation between coupling strength and LF-HRV after RETROICOR. The reduction of coupling strength after RETROICOR needs to be interpreted carefully. The relationship between the BOLD signal and heart rate is a complex one, and cannot be disregarded as purely artefactual. Indeed, direct measures of neural activity in humans show a link 
between heart rate and spontaneous neural firing rate in single- and multi-unit recordings (Kim et al., 2019), as well as with EEG alpha power (de Munck et al., 2008).

\section{Possible functional roles of gastric-brain coupling}

The precise function of the coupling between the BOLD signal and the gastric rhythm in this extended sensory and motor network remains highly speculative at this stage. Indeed, it takes a lot of information to define the function of a given brain region (Genon et al., 2018), let alone a whole network. Much remains to be studied, including notably task-dependency, directionality of the interaction, as well as shared vs. unique contribution of each sub-region of the gastric network. Still, the spatial layout of the gastric network and the involvement of all sensory and motor cortices is puzzling, and calls for further interpretation. In the following, we consider several non-exclusive working hypotheses regarding the potential functional consequences of gastric-brain coupling.

The gastric network could be functionally related to interoception and autonomic processes. Somatosensory, motor, premotor, cingulate motor and insular cortices, which belong to the gastric network, have established roles in interoception and autonomic processes (Critchley et al., 2004; Dum et al., 2016; Levinthal \& Strick, 2020), and the strength of gastric-brain coupling is related to an index of cardiac activity. Given the physiological function of the stomach, a specific link with feeding behavior should be considered. A recent study found an negative correlation between weight loss and power at $0.05 \mathrm{~Hz}$ in gastric network regions across 90 individuals undergoing a weight reduction intervention (Levakov et al., 2020), suggesting a link between energy regulation and brain activity at gastric frequency. Note that the contribution of the cerebellum to the gastric network is compatible with a role in interoception and autonomic processes, since the cerebellum has been associated with activity from different bodily systems, such as cardiovascular (Reis \& Golanov, 1997), respiratory (Xu \& Frazier, 2000, p. 200), as well as in feeding control (Liu et al., 2000; Tataranni et al., 1999; Zhu \& Wang, 2008). Still, the coupling between gastric rhythm and activity in 
early auditory and visual regions is not readily explained by a functional role of the gastric network limited to bodily regulations.

Another view, that might encompass a link with energy regulation, is that gastric-brain coupling is related to arousal, given the anatomical and functional links reviewed above, as well as to the observation that the amplitude of parieto-occipital alpha rhythm is coupled to the gastric rhythm in humans (Richter et al., 2017). Whether gastric-brain coupling correspond to fluctuations of arousal within one gastric cycle, akin to the notion "pulsed arousal" associated with the cardiac cycle (Garfinkel \& Critchley, 2016), or to longer episodes of high or low arousal spanning several gastric cycles, remains to be determined. However, this view does not account for the specific layout of the gastric network. Indeed, why should gastric-related fluctuations of arousal be concentrated in sensory and motor regions and spare most of the transmodal regions typically associated with conceptual, abstract processing (Behrens et al., 2013)?

Finally, gastric-brain coupling might reflect an overall scaffolding mechanism contributing to the organization of large-scale neural activity, involved in the coordination between brain regions to bind information (Fries, 2005; Singer \& Gray, 1995) expressed in different formats and coordinates (Azzalini et al., 2019). The sensory and motor regions of the gastric network contain topographical representations of the body surface, activated by touch, movement or visual perception of body parts (Amiez \& Petrides, 2014; Orlov et al., 2010; Penfield \& Boldrey, 1937), as well as of external space, which is represented in retinotopic coordinates in visual cortices and in tonotopic space in auditory cortices. The gastric rhythm, acting as a common input to all those regions, could facilitate the alignment and coordination of the different coordinate systems in which external information from the senses is expressed - in other words, act as a binding mechanism facilitating between-area communication (Azzalini et al., 2019) - with the stomach delivering different time stamps to different regions in a mechanism reminiscent of traveling waves (X.-J. Wang, 2010). Such a facilitation of inter- 
767

areal communication between sensory and motor regions would be an interesting complement to the known top-down control from cognitive areas to sensory regions.

\section{Conclusion}

770

The multiple cortical areas of the human brain have long been thought be organized in an ascending hierarchy (Felleman \& Van Essen, 1991) composed of relatively independent modules corresponding to each sensory modality converging onto higher-order, transmodal regions (Mesulam, 1998). While this view has been refined (see e.g., (Markov et al., 2013; Young, 1992), it is 775 still much present in the narrative of large scale brain organization, and fits with the classical 776 parcellation in distinct resting-state networks for different modalities (Yeo et al., 2011). Our findings 777 show that regions considered to be mostly independent are actually all tightly linked through delayed 778 functional connectivity with the stomach. The monitoring of visceral inputs should thus be integrated 779 into current views of the cortical hierarchy. 
781

782

\section{References}

Addis, D. R., Wong, A. T., \& Schacter, D. L. (2007). Remembering the past and imagining the future : Common and distinct neural substrates during event construction and elaboration. Neuropsychologia, 45(7), 1363-1377. https://doi.org/10.1016/j.neuropsychologia.2006.10.016

Allen, M., Poggiali, D., Whitaker, K., Marshall, T. R., \& Kievit, R. A. (2019). Raincloud plots : A multiplatform tool for robust data visualization. Wellcome Open Research, 4, 63. https://doi.org/10.12688/wellcomeopenres.15191.1

Amassian, V. E. (1951). Cortical representation of visceral afferents. Journal of Neurophysiology, 14(6), 433-444.

Amiez, C., \& Petrides, M. (2014). Neuroimaging Evidence of the Anatomo-Functional Organization of the Human Cingulate Motor Areas. Cerebral Cortex, 24(3), 563-578. https://doi.org/10.1093/cercor/bhs329

Andrews-Hanna, J. R., Smallwood, J., \& Spreng, R. N. (2014). The default network and self-generated thought : Component processes, dynamic control, and clinical relevance. Annals of the New York Academy of Sciences, 1316(1), 29-52. https://doi.org/10.1111/nyas.12360

Ashburner, J., Friston, K. J., \& others. (1999). Nonlinear spatial normalization using basis functions. Human brain mapping, 7(4), 254-266.

Azzalini, D., Rebollo, I., \& Tallon-Baudry, C. (2019). Visceral Signals Shape Brain Dynamics and Cognition. Trends in Cognitive Sciences. https://doi.org/10.1016/j.tics.2019.03.007

Barrett, L. F., \& Simmons, W. K. (2015). Interoceptive predictions in the brain. Nature reviews neuroscience, 16(7), 419-429.

Bastos, A. M., \& Schoffelen, J.-M. (2016). A Tutorial Review of Functional Connectivity Analysis Methods and Their Interpretational Pitfalls. Frontiers in Systems Neuroscience, 9:175. https://doi.org/10.3389/fnsys.2015.00175 
Behrens, T. E. J., Fox, P., Laird, A., \& Smith, S. M. (2013). What is the most interesting part of the brain? Trends in cognitive sciences, 17(1), 2-4. https://doi.org/10.1016/j.tics.2012.10.010

Biswal, B., Zerrin Yetkin, F., Haughton, V. M., \& Hyde, J. S. (1995). Functional connectivity in the motor cortex of resting human brain using echo-planar mri. Magnetic resonance in medicine, 34(4), 537-541.

Cao, J., Lu, K.-H., Oleson, S. T., Phillips, R. J., Jaffey, D., Hendren, C. L., Powley, T. L., \& Liu, Z. (2019). Gastric stimulation drives fast BOLD responses of neural origin. Neurolmage, 197, 200-211. https://doi.org/10.1016/j.neuroimage.2019.04.064

Cappe, C., \& Barone, P. (2005). Heteromodal connections supporting multisensory integration at low levels of cortical processing in the monkey. The European Journal of Neuroscience, 22(11), 2886-2902. https://doi.org/10.1111/j.1460-9568.2005.04462.x

Catani, M. (2017). A little man of some importance. Brain, 140(11), 3055-3061.

Cechetto, D. F., \& Saper, C. B. (1987). Evidence for a viscerotopic sensory representation in the cortex and thalamus in the rat. Journal of Comparative Neurology, 262(1), 27-45.

Chang, C., Metzger, C. D., Glover, G. H., Duyn, J. H., Heinze, H.-J., \& Walter, M. (2013). Association between heart rate variability and fluctuations in resting-state functional connectivity. Neurolmage, 68, 93-104. https://doi.org/10.1016/j.neuroimage.2012.11.038

Choe, A. S., Tang, B., Smith, K. R., Honari, H., Lindquist, M. A., Caffo, B. S., \& Pekar, J. J. (2020). Phaselocking of resting-state brain networks with the gastric basal electrical rhythm. BioRxiv, 2020.10.06.328054. https://doi.org/10.1101/2020.10.06.328054

Coen, S. J., Hobson, A. R., \& Aziz, Q. (2012). Chapter 23-Processing of Gastrointestinal Sensory Signals in the Brain. In L. R. Johnson, F. K. Ghishan, J. D. Kaunitz, J. L. Merchant, H. M. Said, \& J. D. Wood (Éds.), Physiology of the Gastrointestinal Tract (Fifth Edition) (p. 689-702). Academic Press. https://doi.org/10.1016/B978-0-12-382026-6.00023-3 
Critchley, H. D., Wiens, S., Rotshtein, P., Öhman, A., \& Dolan, R. J. (2004). Neural systems supporting interoceptive awareness. Nature Neuroscience, 7(2), 189-195.

https://doi.org/10.1038/nn1176

Cruz, M. T., Murphy, E. C., Sahibzada, N., Verbalis, J. G., \& Gillis, R. A. (2007). A reevaluation of the effects of stimulation of the dorsal motor nucleus of the vagus on gastric motility in the rat. American Journal of Physiology-Regulatory, Integrative and Comparative Physiology, 292(1), R291-R307. https://doi.org/10.1152/ajpregu.00863.2005

de Munck, J. C., Gonçalves, S. I., Faes, Th. J. C., Kuijer, J. P. A., Pouwels, P. J. W., Heethaar, R. M., \& Lopes da Silva, F. H. (2008). A study of the brain's resting state based on alpha band power, heart rate and fMRI. Neurolmage, 42(1), 112-121. https://doi.org/10.1016/j.neuroimage.2008.04.244

Delorme, A., \& Makeig, S. (2004). EEGLAB : An open source toolbox for analysis of single-trial EEG dynamics including independent component analysis. Journal of Neuroscience Methods, 134(1), 9-21. https://doi.org/10.1016/j.jneumeth.2003.10.009

Desikan, R. S., Ségonne, F., Fischl, B., Quinn, B. T., Dickerson, B. C., Blacker, D., Buckner, R. L., Dale, A. M., Maguire, R. P., Hyman, B. T., Albert, M. S., \& Killiany, R. J. (2006). An automated labeling system for subdividing the human cerebral cortex on MRI scans into gyral based regions of interest. Neurolmage, 31(3), 968-980. https://doi.org/10.1016/j.neuroimage.2006.01.021

Domschke, K., Stevens, S., Pfleiderer, B., \& Gerlach, A. L. (2010). Interoceptive sensitivity in anxiety and anxiety disorders : An overview and integration of neurobiological findings. Clinical Psychology Review, 30(1), 1-11. https://doi.org/10.1016/j.cpr.2009.08.008

Dum, R. P., Levinthal, D. J., \& Strick, P. L. (2009). The Spinothalamic System Targets Motor and Sensory Areas in the Cerebral Cortex of Monkeys. Journal of Neuroscience, 29(45), 14223-14235. https://doi.org/10.1523/JNEUROSCI.3398-09.2009 
Dum, R. P., Levinthal, D. J., \& Strick, P. L. (2016). Motor, cognitive, and affective areas of the cerebral cortex influence the adrenal medulla. Proceedings of the National Academy of Sciences, 113(35), 9922-9927. https://doi.org/10.1073/pnas.1605044113

Elam, M., Thorén, P., \& Svensson, T. H. (1986). Locus coeruleus neurons and sympathetic nerves : Activation by visceral afferents. Brain Research, 375(1), 117-125. https://doi.org/10.1016/0006-8993(86)90964-9

Erişir, A., Van Horn, S. C., \& Sherman, S. M. (1997). Relative numbers of cortical and brainstem inputs to the lateral geniculate nucleus. Proceedings of the National Academy of Sciences, 94(4), 1517-1520. https://doi.org/10.1073/pnas.94.4.1517

Felleman, D. J., \& Van Essen, D. C. (1991). Distributed hierarchical processing in the primate cerebral cortex. Cerebral Cortex (New York, N.Y.: 1991), 1(1), 1-47. https://doi.org/10.1093/cercor/1.1.1

Fries, P. (2005). A mechanism for cognitive dynamics : Neuronal communication through neuronal coherence. Trends in Cognitive Sciences, 9(10), 474-480. https://doi.org/10.1016/j.tics.2005.08.011

Friston, K. J., Ashburner, J., Frith, C. D., Poline, J.-B., Heather, J. D., \& Frackowiak, R. S. J. (1995). Spatial registration and normalization of images. Human Brain Mapping, 3(3), 165-189. https://doi.org/10.1002/hbm.460030303

Garfinkel, S. N., \& Critchley, H. D. (2016). Threat and the Body : How the Heart Supports Fear Processing. Trends in Cognitive Sciences, 20(1), 34-46. https://doi.org/10.1016/j.tics.2015.10.005

Genon, S., Reid, A., Li, H., Fan, L., Müller, V. I., Cieslik, E. C., Hoffstaedter, F., Langner, R., Grefkes, C., Laird, A. R., Fox, P. T., Jiang, T., Amunts, K., \& Eickhoff, S. B. (2018). The heterogeneity of the left dorsal premotor cortex evidenced by multimodal connectivity-based parcellation and functional characterization. Neurolmage, 170, 400-411. https://doi.org/10.1016/j.neuroimage.2017.02.034 
Glasser, M. F., Coalson, T. S., Robinson, E. C., Hacker, C. D., Harwell, J., Yacoub, E., Ugurbil, K., Andersson, J., Beckmann, C. F., Jenkinson, M., Smith, S. M., \& Essen, D. C. V. (2016). A multimodal parcellation of human cerebral cortex. Nature, 536(7615), 171. https://doi.org/10.1038/nature18933

Glover, G. H., Li, T. Q., \& Ress, D. (2000). Image-based method for retrospective correction of physiological motion effects in fMRI : RETROICOR. Magnetic Resonance in Medicine, 44(1), 162-167.

Goldstein, D. S., Bentho, O., Park, M.-Y., \& Sharabi, Y. (2011). LF power of heart rate variability is not a measure of cardiac sympathetic tone but may be a measure of modulation of cardiac autonomic outflows by baroreflexes. Experimental physiology, 96(12), 1255-1261. https://doi.org/10.1113/expphysiol.2010.056259

Huntenburg, J. M., Bazin, P.-L., Goulas, A., Tardif, C. L., Villringer, A., \& Margulies, D. S. (2017). A Systematic Relationship Between Functional Connectivity and Intracortical Myelin in the Human Cerebral Cortex. Cerebral Cortex (New York, N.Y.: 1991), 27(2), 981-997. https://doi.org/10.1093/cercor/bhx030

Huntenburg, J. M., Bazin, P.-L., \& Margulies, D. S. (2018). Large-Scale Gradients in Human Cortical Organization. Trends in Cognitive Sciences, 22(1), 21-31. https://doi.org/10.1016/j.tics.2017.11.002

lannetti, G. D., Niazy, R. K., Wise, R. G., Jezzard, P., Brooks, J. C. W., Zambreanu, L., Vennart, W., Matthews, P. M., \& Tracey, I. (2005). Simultaneous recording of laser-evoked brain potentials and continuous, high-field functional magnetic resonance imaging in humans. Neurolmage, 28(3), 708-719. https://doi.org/10.1016/j.neuroimage.2005.06.060

Jasmin, K., Lima, C. F., \& Scott, S. K. (2019). Understanding rostral-caudal auditory cortex contributions to auditory perception. Nature Reviews Neuroscience, 20(7), 425-434. https://doi.org/10.1038/s41583-019-0160-2 
Kasper, L., Bollmann, S., Diaconescu, A. O., Hutton, C., Heinzle, J., Iglesias, S., Hauser, T. U., Sebold, M., Manjaly, Z.-M., Pruessmann, K. P., \& Stephan, K. E. (2017). The PhysIO Toolbox for Modeling Physiological Noise in fMRI Data. Journal of Neuroscience Methods, 276, 56-72. https://doi.org/10.1016/j.jneumeth.2016.10.019

Kass, R. E., \& Raftery, A. E. (2012). Bayes Factors. Journal of the American Statistical Association, 90(430), 773-795.

Kern, M., Aertsen, A., Schulze-Bonhage, A., \& Ball, T. (2013). Heart cycle-related effects on eventrelated potentials, spectral power changes, and connectivity patterns in the human ECoG. Neurolmage, 81, 178-190. https://doi.org/10.1016/j.neuroimage.2013.05.042

Kern, M. K., \& Shaker, R. (2002). Cerebral cortical registration of subliminal visceral stimulation. Gastroenterology, 122(2), 290-298. https://doi.org/10.1053/gast.2002.30989

Kim, K., Ladenbauer, J., Babo-Rebelo, M., Buot, A., Lehongre, K., Adam, C., Hasboun, D., Lambrecq, V., Navarro, V., Ostojic, S., \& Tallon-Baudry, C. (2019). Resting-State Neural Firing Rate Is Linked to Cardiac-Cycle Duration in the Human Cingulate and Parahippocampal Cortices. Journal of Neuroscience, 39(19), 3676-3686. https://doi.org/10.1523/JNEUROSCI.2291-18.2019

Koch, K. L., \& Robert M. Stern. (2004). Handbook of Electrogastrography (Oxford University Press).

Krienen, F. M., Yeo, B. T. T., Ge, T., Buckner, R. L., \& Sherwood, C. C. (2016). Transcriptional profiles of supragranular-enriched genes associate with corticocortical network architecture in the human brain. Proceedings of the National Academy of Sciences of the United States of America, 113(4), E469-478. https://doi.org/10.1073/pnas.1510903113

Kukorelli, T., \& Juhász, G. (1977). Sleep induced by intestinal stimulation in cats. Physiology \& Behavior, 19(3), 355-358.

Lachaux, J.-P., Rodriguez, E., Martinerie, J., Varela, F. J., \& others. (1999). Measuring phase synchrony in brain signals. Human brain mapping, 8(4), 194-208.

Levakov, G., Kaplan, A., Yaskolka Meir, A., Rinott, E., Tsaban, G., Zelicha, H., Meiran, N., Shelef, I., Shai, I., \& Avidan, G. (2020). Neural correlates of future weight loss reveal a possible role for 
brain-gastric interactions. Neurolmage, 224, 117403.

https://doi.org/10.1016/j.neuroimage.2020.117403

Levinthal, D. J., \& Strick, P. L. (2020). Multiple areas of the cerebral cortex influence the stomach. Proceedings of the National Academy of Sciences, 117(23), 13078-13083. https://doi.org/10.1073/pnas.2002737117

Liu, Y., Gao, J.-H., Liu, H.-L., \& Fox, P. T. (2000). The temporal response of the brain after eating revealed by functional MRI. Nature, 405(6790), 1058-1062. https://doi.org/10.1038/35016590

Margulies, D. S., Ghosh, S. S., Goulas, A., Falkiewicz, M., Huntenburg, J. M., Langs, G., Bezgin, G., Eickhoff, S. B., Castellanos, F. X., Petrides, M., Jefferies, E., \& Smallwood, J. (2016). Situating the default-mode network along a principal gradient of macroscale cortical organization. Proceedings of the National Academy of Sciences, 113(44), 12574-12579. https://doi.org/10.1073/pnas.1608282113

Maris, E., \& Oostenveld, R. (2007). Nonparametric statistical testing of EEG- and MEG-data. Journal of Neuroscience Methods, 164(1), 177-190. https://doi.org/10.1016/j.jneumeth.2007.03.024

Markov, N. T., Ercsey-Ravasz, M., Van Essen, D. C., Knoblauch, K., Toroczkai, Z., \& Kennedy, H. (2013). Cortical high-density counterstream architectures. Science (New York, N.Y.), 342(6158), 1238406. https://doi.org/10.1126/science.1238406

Mesulam, M. M. (1998). From sensation to cognition. Brain, 121(6), 1013-1052. https://doi.org/10.1093/brain/121.6.1013

Niazy, R. K., Beckmann, C. F., lannetti, G. D., Brady, J. M., \& Smith, S. M. (2005). Removal of FMRI environment artifacts from EEG data using optimal basis sets. Neurolmage, 28(3), 720-737. https://doi.org/10.1016/j.neuroimage.2005.06.067

Oostenveld, R., Fries, P., Maris, E., \& Schoffelen, J.-M. (2010). FieldTrip : Open Source Software for Advanced Analysis of MEG, EEG, and Invasive Electrophysiological Data. Computational Intelligence and Neuroscience, 2011. https://doi.org/10.1155/2011/156869 
Orlov, T., Makin, T. R., \& Zohary, E. (2010). Topographic representation of the human body in the occipitotemporal cortex. Neuron, 68(3), 586-600. https://doi.org/10.1016/j.neuron.2010.09.032

Penfield, W., \& Boldrey, E. (1937). Somatic motor and sensory representation in the cerebral cortex of man as studied by electrical stimulation. Brain, 60(4), 389-443.

Petzschner, F. H., Garfinkel, S. N., Paulus, M. P., Koch, C., \& Khalsa, S. S. (2021). Computational models of interoception and body regulation. Trends in neurosciences, 44(1), 63-76.

Power, J. D., Barnes, K. A., Snyder, A. Z., Schlaggar, B. L., \& Petersen, S. E. (2012). Spurious but systematic correlations in functional connectivity MRI networks arise from subject motion. Neurolmage, 59(3), 2142-2154. https://doi.org/10.1016/j.neuroimage.2011.10.018

Power, J. D., Schlaggar, B. L., \& Petersen, S. E. (2014). Studying brain organization via spontaneous fMRI signal. Neuron, 84(4), 681-696. https://doi.org/10.1016/j.neuron.2014.09.007

Rebollo, I., Devauchelle, A.-D., Béranger, B., \& Tallon-Baudry, C. (2018). Stomach-brain synchrony reveals a novel, delayed-connectivity resting-state network in humans. ELife, 7, e33321. https://doi.org/10.7554/eLife.33321

Rebollo, I., Wolpert, N., \& Tallon-Baudry, C. (2021). Brain-stomach coupling : Anatomy, functions, and future avenues of research. Current Opinion in Biomedical Engineering, 100270. https://doi.org/10.1016/j.cobme.2021.100270

Reis, D. J., \& Golanov, E. V. (1997). Autonomic and vasomotor regulation. International Review of Neurobiology, 41, 121-149.

Reyes del Paso, G. A., Langewitz, W., Mulder, L. J. M., van Roon, A., \& Duschek, S. (2013). The utility of low frequency heart rate variability as an index of sympathetic cardiac tone : A review with emphasis on a reanalysis of previous studies. Psychophysiology, 50(5), 477-487. https://doi.org/10.1111/psyp.12027

Richter, C. G., Babo-Rebelo, M., Schwartz, D., \& Tallon-Baudry, C. (2017). Phase-amplitude coupling at the organism level : The amplitude of spontaneous alpha rhythm fluctuations varies with 
the phase of the infra-slow gastric basal rhythm. Neurolmage, 146, 951-958.

https://doi.org/10.1016/j.neuroimage.2016.08.043

Rinaman, L., \& Schwartz, G. (2004). Anterograde Transneuronal Viral Tracing of Central Viscerosensory Pathways in Rats. Journal of Neuroscience, 24(11), 2782-2786. https://doi.org/10.1523/JNEUROSCI.5329-03.2004

Ripley, B., Venables, B., Bates, D. M., Hornik, K., Gebhardt, A., Firth, D., \& Ripley, M. B. (2013). Package 'mass'. Cran R.

Rodenkirch, C., Liu, Y., Schriver, B. J., \& Wang, Q. (2019). Locus coeruleus activation enhances thalamic feature selectivity via norepinephrine regulation of intrathalamic circuit dynamics. Nature Neuroscience, 22(1), 120-133. https://doi.org/10.1038/s41593-018-0283-1

Rouder, J. N., \& Morey, R. D. (2011). A Bayes factor meta-analysis of Bem's ESP claim. Psychonomic Bulletin \& Review, 18(4), 682-689. https://doi.org/10.3758/s13423-011-0088-7

Saito, K., Kanazawa, M., \& Fukudo, S. (2002). Colorectal distention induces hippocampal noradrenaline release in rats : An in vivo microdialysis study. Brain Research, 947(1), 146-149. https://doi.org/10.1016/S0006-8993(02)03007-X

Sanders, K. M., Ward, S. M., \& Koh, S. D. (2014). Interstitial cells : Regulators of smooth muscle function. Physiological Reviews, 94(3), 859-907. https://doi.org/10.1152/physrev.00037.2013

Saper, C. B., \& Loewy, A. D. (1980). Efferent connections of the parabrachial nucleus in the rat. Brain Research, 197(2), 291-317.

Shmueli, K., van Gelderen, P., de Zwart, J. A., Horovitz, S. G., Fukunaga, M., Jansma, J. M., \& Duyn, J. H. (2007). Low-frequency fluctuations in the cardiac rate as a source of variance in the resting-state fMRI BOLD signal. Neurolmage, 38(2), 306-320. https://doi.org/10.1016/j.neuroimage.2007.07.037

Shokri-Kojori, E., Tomasi, D., \& Volkow, N. D. (2018). An Autonomic Network : Synchrony Between Slow Rhythms of Pulse and Brain Resting State Is Associated with Personality and Emotions. 
1008

1009

1010

1011

1012

1013

1014

1015

1016

1018

1019

1020

1021

1022

1023

1024

1025

1026

1028

1029

1030

1031

1032

1033
Cerebral Cortex (New York, N.Y.: 1991), 28(9), 3356-3371.

https://doi.org/10.1093/cercor/bhy144

Singer, W., \& Gray, C. M. (1995). Visual Feature Integration and the Temporal Correlation Hypothesis. Annual Review of Neuroscience, 18(1), 555-586.

https://doi.org/10.1146/annurev.ne.18.030195.003011

Spielberger, C. D., Gorsuch, R. L., Lushene, R., Vagg, P. R., \& Jacobs, G. A. (1983). Consulting Psychologists Press; Palo Alto, CA : 1983. Manual for the state-trait anxiety inventory.

Sveshnikov, D. S., Smirnov, V. M., Myasnikov, I. L., \& Kuchuk, A. V. (2012). Study of the Nature of Sympathetic Trunk Nerve Fibers Enhancing Gastric Motility. Bulletin of Experimental Biology and Medicine, 152(3), 283-285. https://doi.org/10.1007/s10517-012-1508-z

Tataranni, P. A., Gautier, J.-F., Chen, K., Uecker, A., Bandy, D., Salbe, A. D., Pratley, R. E., Lawson, M., Reiman, E. M., \& Ravussin, E. (1999). Neuroanatomical correlates of hunger and satiation in humans using positron emission tomography. Proceedings of the National Academy of Sciences, 96(8), 4569-4574. https://doi.org/10.1073/pnas.96.8.4569

Tort, A. B. L., Brankačk, J., \& Draguhn, A. (2018). Respiration-Entrained Brain Rhythms Are Global but Often Overlooked. Trends in Neurosciences, 41(4), 186-197. https://doi.org/10.1016/j.tins.2018.01.007

Travagli, R. A., Hermann, G. E., Browning, K. N., \& Rogers, R. C. (2006). Brainstem circuits regulating gastric function. Annu. Rev. Physiol., 68, 279-305.

Uhlrich, D. J., Cucchiaro, J. B., \& Sherman, S. M. (1988). The projection of individual axons from the parabrachial region of the brain stem to the dorsal lateral geniculate nucleus in the cat. The Journal of Neuroscience: The Official Journal of the Society for Neuroscience, 8(12), 4565-4575.

Van Oudenhove, I., Vandenberghe, J., Dupont, P., Geeraerts, B., Vos, R., Bormans, G., Van Laere, K., Fischler, B., Demyttenaere, K., Janssens, J., \& Tack, J. (2009). Cortical deactivations during gastric fundus distension in health : Visceral pain-specific response or attenuation of 'default 
1034

1035

1036

1037

1038

1039

1040

1041

1042

1043

1044

1045

1046

1047

1048

1049

1050

1051

1052

1053

1054

1055

1056

1057

1058

1059

mode' brain function? $\mathrm{A} \mathrm{H}_{2}{ }^{15}$ O-PET study. Neurogastroenterology \& Motility, 21(3), 259-271. https://doi.org/10.1111/j.1365-2982.2008.01196.x

Wagenmakers, E.-J. (2007). A practical solution to the pervasive problems ofp values. Psychonomic Bulletin \& Review, 14(5), 779-804. https://doi.org/10.3758/BF03194105

Wang, X.-J. (2010). Neurophysiological and Computational Principles of Cortical Rhythms in Cognition. Physiological Reviews, 90(3), 1195-1268.

https://doi.org/10.1152/physrev.00035.2008

Wang, Z., Bradesi, S., Maarek, J.-M. I., Lee, K., Winchester, W. J., Mayer, E. A., \& Holschneider, D. P. (2008). Regional brain activation in conscious, nonrestrained rats in response to noxious visceral stimulation. PAIN, 138(1), 233-243. https://doi.org/10.1016/j.pain.2008.04.018

Wetzels, R., \& Wagenmakers, E.-J. (2012). A default Bayesian hypothesis test for correlations and partial correlations. Psychonomic Bulletin \& Review, 19(6), 1057-1064. https://doi.org/10.3758/s13423-012-0295-x

Wolpert, N., Rebollo, I., \& Tallon-Baudry, C. (2020). Electrogastrography for psychophysiological research : Practical considerations, analysis pipeline, and normative data in a large sample. Psychophysiology, e13599. https://doi.org/10.1111/psyp.13599

Wu, J., Ngo, G. H., Greve, D., Li, J., He, T., Fischl, B., Eickhoff, S. B., \& Yeo, B. T. T. (2018). Accurate nonlinear mapping between MNI volumetric and FreeSurfer surface coordinate systems. Human Brain Mapping. https://doi.org/10.1002/hbm.24213

Xu, F., \& Frazier, D. T. (2000). Modulation of respiratory motor output by cerebellar deep nuclei in the rat. Journal of Applied Physiology (Bethesda, Md.: 1985), 89(3), 996-1004. https://doi.org/10.1152/jappl.2000.89.3.996

Yeo, B. T. T., Krienen, F. M., Sepulcre, J., Sabuncu, M. R., Lashkari, D., Hollinshead, M., Roffman, J. L., Smoller, J. W., Zöllei, L., Polimeni, J. R., Fischl, B., Liu, H., \& Buckner, R. L. (2011). The organization of the human cerebral cortex estimated by intrinsic functional connectivity. Journal of Neurophysiology, 106(3), 1125-1165. https://doi.org/10.1152/jn.00338.2011 
1060 1061 1062 1063 1064 1065 1066 1067 1068 1069

1070 1071
Young, M. P. (1992). Objective analysis of the topological organization of the primate cortical visual system. Nature, 358(6382), 152-155. https://doi.org/10.1038/358152a0

Yu, X., Tu, L., Lei, P., Song, J., Xu, H., \& Hou, X. (2014). Antiemesis effect and brain fMRI response of gastric electrical stimulation with different parameters in dogs. Neurogastroenterology \& Motility, 26(7), 1049-1056. https://doi.org/10.1111/nmo.12362

Zhu, J.-N., \& Wang, J.-J. (2008). The Cerebellum in Feeding Control : Possible Function and Mechanism. Cellular and Molecular Neurobiology, 28(4), 469-478. https://doi.org/10.1007/s10571-007-9236-z 
1072 Figure legends

1073

1074

1075

1076

1078

1079

1080

1081

1082

1083

1084

1085

1086

1088

1089

1090

1091

1092

1093

1094

1095

1096

Figure 1: The gastric network and resting state networks. A- Effect sizes (Cohen's d) of gastric-BOLD coupling are plotted in orange in regions significantly phase synchronized to the gastric rhythm ( $n=63$, voxel-level threshold $=0.05$ one-sided and cluster significance $<0.025$ one-sided, intrinsically corrected for multiple comparisons), overlaid on top of the cortical parcellation in seven RSNs proposed by (Yeo et al., 2011), color codes as in E. The gastric network also comprises left striatum (B), bilateral thalamus (C) and cerebellum (D). E- Percentage of the gastric network in each of the brain's RSNs (left), and average effect size across significant voxels within each network (right) for the whole sample (top), sample 1 ( $n=29$, middle), and sample 2 ( $n=34$, bottom). The standard error in effect size was obtained by a bootstrapping procedure. Note that in the Yeo et al 2009 parcellation, subcortical refers to thalamus and striatum but does not include cerebellum. F- Average coupling in the gastric networks across participants, green sample 1, blue sample 2. G- twenty-five regions from (Glasser et al., 2016) parcellation showing the largest effect sizes averaged across hemispheres. Arrowheads depict effect sizes for left $(<)$ and right $(>)$ hemispheres. Abbreviations: RSN, resting state network; Somatomot, somato-motor-auditory. Tables in extended data 1-1 and 1-2 contain the tvalues peak coordinates in MNI space and the effect sizes in each region of the Glasser (2017) parcellation respectively.

Figure 2: The gastric network and cortical gradients of functional connectivity. A- Density plot depicting the distribution of all cortical voxels along the first two gradients of functional connectivity described by (Margulies et al., 2016). The first gradient (y-axis), runs from unimodal (negative values, sensory or motor regions) to transmodal regions (positive values). The second gradient (x-axis) runs from visual (positive values) to somato-motor and auditory regions (negative values). The color scale depicts the logarithm of the number of voxels. The projection of the probability density on the first and second gradients are shown in red and blue respectively. B- Density plot of gastric network 
voxels on the first two gradients of functional connectivity. Gastric network voxels are located in the unimodal extremes of the two gradients. The projection of the probability density on the first and second gradients are shown in red and blue respectively. C- Percentage of all brain voxels that belong to the gastric network (orange) for each of the two gradients, computed on one hundred equidistant bins. The black circles depict the distribution of chance level overlap obtained by reallocating randomly the spatial position of gastric network voxels in the cortex. Gradients downloaded from https://identifiers.org/neurovault.collection:1598

Figure 3: Gastric network in central and mid-cingulate regions. A- Effect sizes in the gastric network in central (top) and mid-cingulate regions (bottom) displayed on the left and right inflated surfaces and overlaid with the corresponding regions of Glasser et al 2016 parcellation. Green, primary somatosensory; Blue, motor and premotor regions; Pink, area 55b; Violet, non-motor cingulate regions; Cyan, area 5 and its subdivisions. B- Percentage of each region overlapping with the gastric network. C- Effect sizes of gastric-BOLD coupling in voxels belonging to the gastric network split by regions. Abbreviations as in text and extended data in figure 1-2.

Figure 4: Gastric network in and around lateral sulcus. A- Effect sizes in the gastric network in and around left and right lateral sulci, displayed on inflated cortical surfaces and overlaid with the corresponding regions of the Glasser 2016 parcellation. Green, secondary somatosensory cortex and area PFcm; Blue, frontal operculum; Cyan, area 43; Dark violet, early auditory; Light Violet, auditory association; Pink, Insula proper. B- Percentage of each region overlapping with the gastric network. C- Effect sizes of gastric-BOLD coupling in voxels belonging to the gastric network split by regions. Abbreviations as in text and extended data in figure 1-2.

Figure 5: Gastric network in posterior regions. A- Medial (top) and lateral (bottom) views of effect sizes in the gastric network in left and right posterior regions, displayed on inflated cortical surfaces and overlaid with the corresponding regions of Glasser 2016 parcellation. Dark green, early visual; 
Blue, occipito-parietal sulcus; Violet, retrosplenial and posterior cingulate cortices; Yellow, ventral precuneus; Light Green, dorsal precuneus; Black, temporo-parietal-occipital junction; Pink, lateral occipital and fusiform. B- Percentage of voxels belonging to the gastric network, in each region. CEffect sizes of gastric-BOLD coupling in voxels belonging to the gastric network split by regions. Abbreviations as in text and extended data in figure 1-2.

Figure 6: Gastric network in prefrontal and lateral temporal. A- Frontal (top) and lateral (bottom) views of effect sizes in the gastric network along left and right prefrontal and lateral temporal regions, displayed in inflated cortical surfaces along with the corresponding regions of Glasser 2016 parcellation. Light Green, lateral prefrontal cortex; Yellow, orbitofrontal cortex; Blue, inferior frontal gyrus; Pink, superior temporal sulcus. B- Percentage of each region belonging to the gastric network. C- Effect sizes of gastric-BOLD coupling in voxels belonging to the gastric network split by regions. Abbreviations as in text and extended data in figure 1-2.

Figure 7. Association between coupling strength and autonomic activity A- Inter-beat intervals (IBI), or time difference between each heart-beat, are used to build time series of heart rate variability. Reproduced from (Azzalini et al., 2019). B. The power spectrum of heart-rate variability, displaying a prominent peak in the low-frequency band $(\mathrm{LF}, 0.05-0.15 \mathrm{~Hz})$, reflecting both sympathetic and parasympathetic activity, and in the high-frequency band, $(\mathrm{HF}, 0.16-0.25 \mathrm{~Hz})$, reflecting parasympathetic activity. C- A robust linear regression shows a relationship between the average coupling strength in the gastric network and the LF-HRV $\left(n=52, t(50)=3.76\right.$, robust regression, $r^{2}=$ 0.22 , uncorrected $p=0.0004$, Bonferroni corrected $p=0.0048)$. D- Percentage of the gastric network in each of the brain's RSNs before (top) and after (bottom) correcting for cardiac timing using RETROICOR (Glover et al., 2000). E- Average coupling strength in the gastric network across 52 participants before and after RETROICOR. 

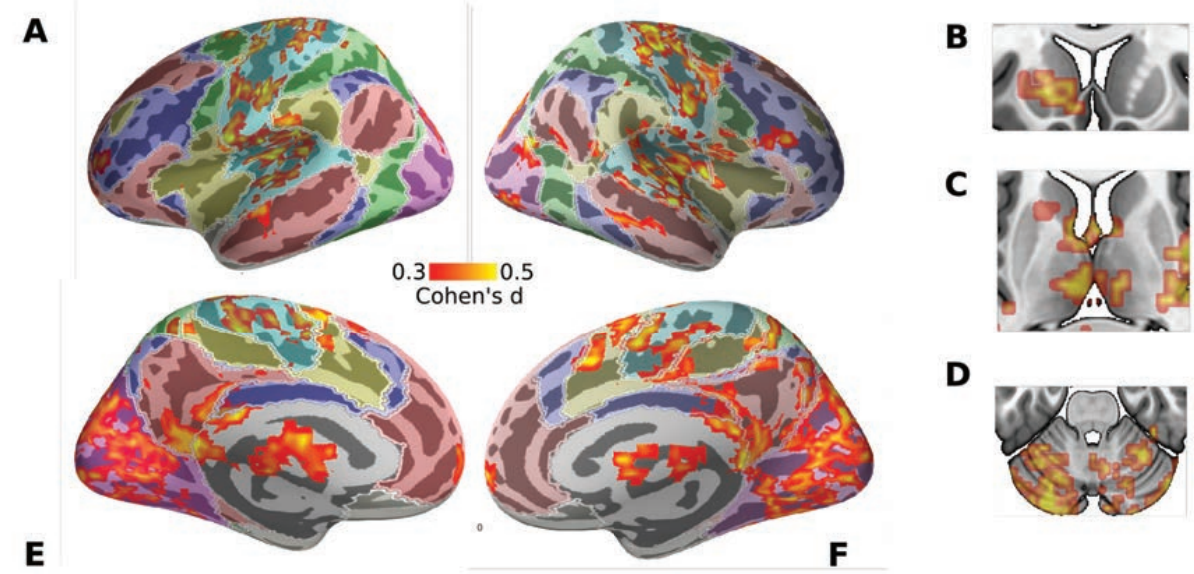

D
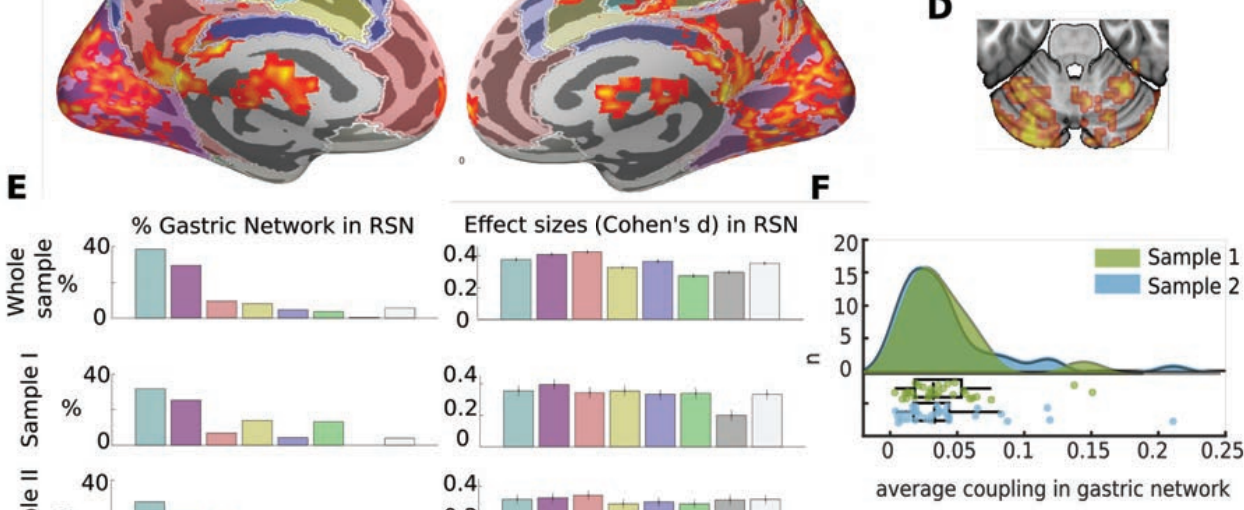

G

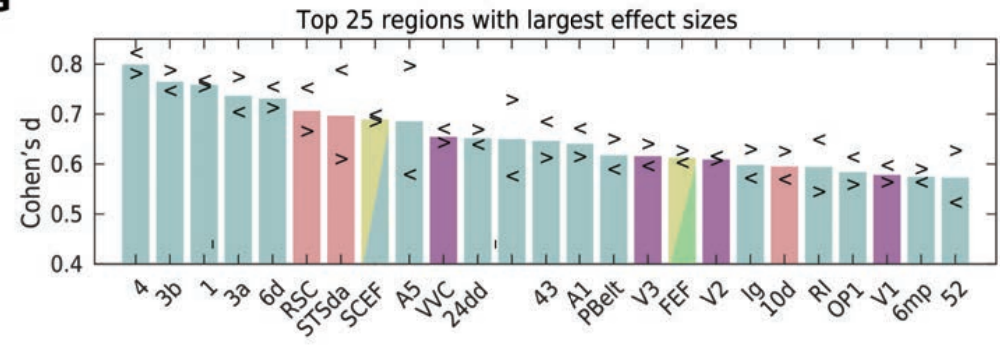




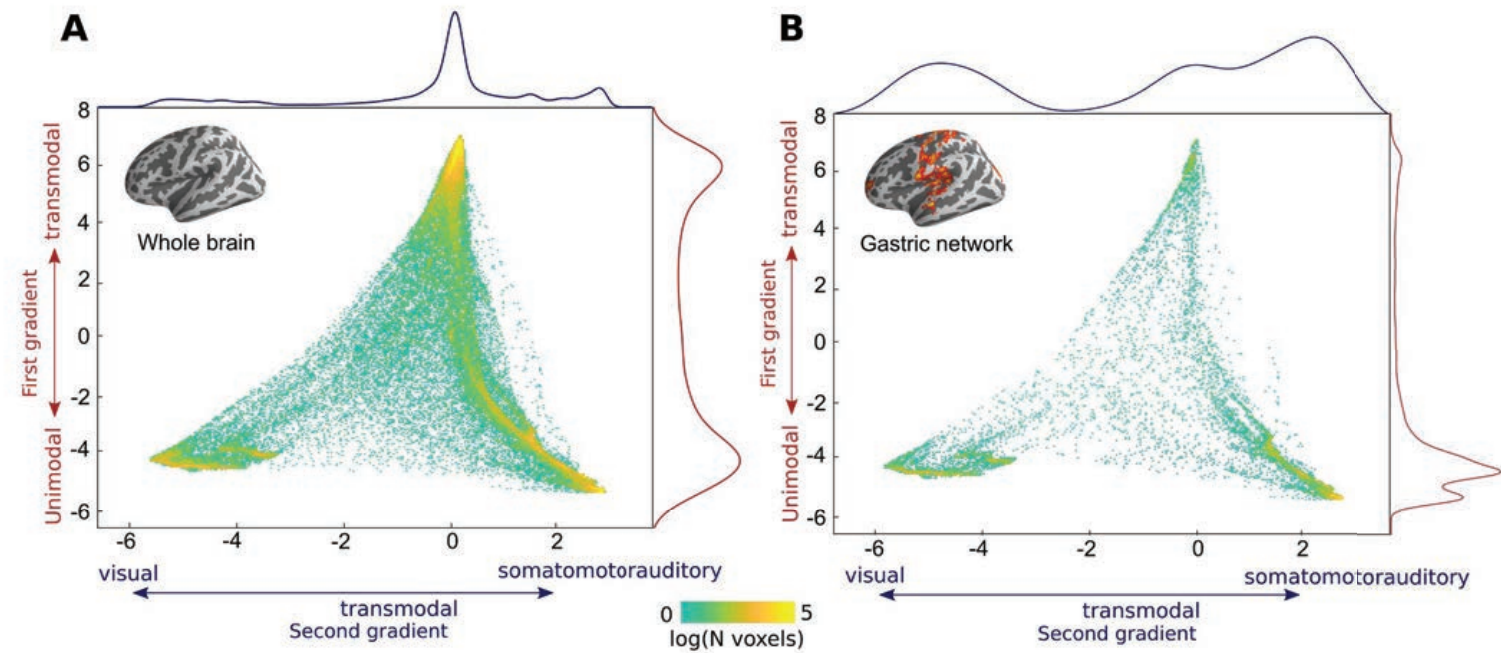

C

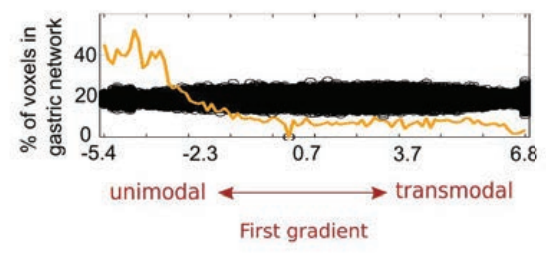

0 - surrogate

- empirical

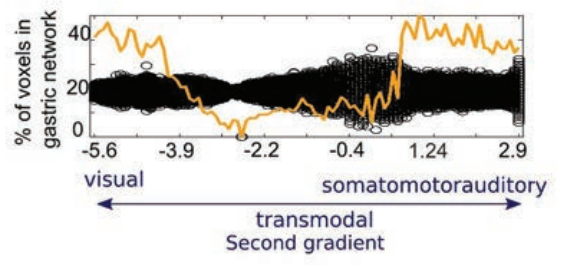



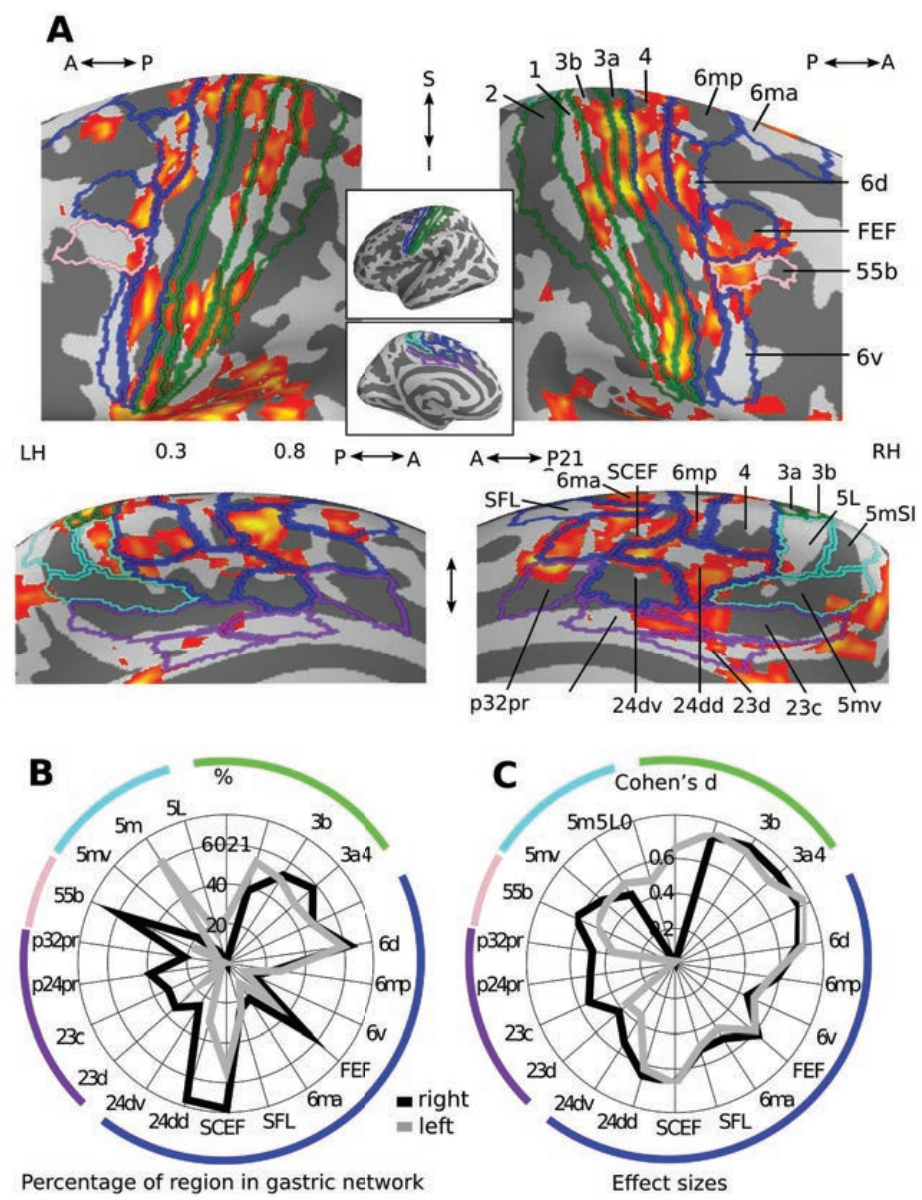

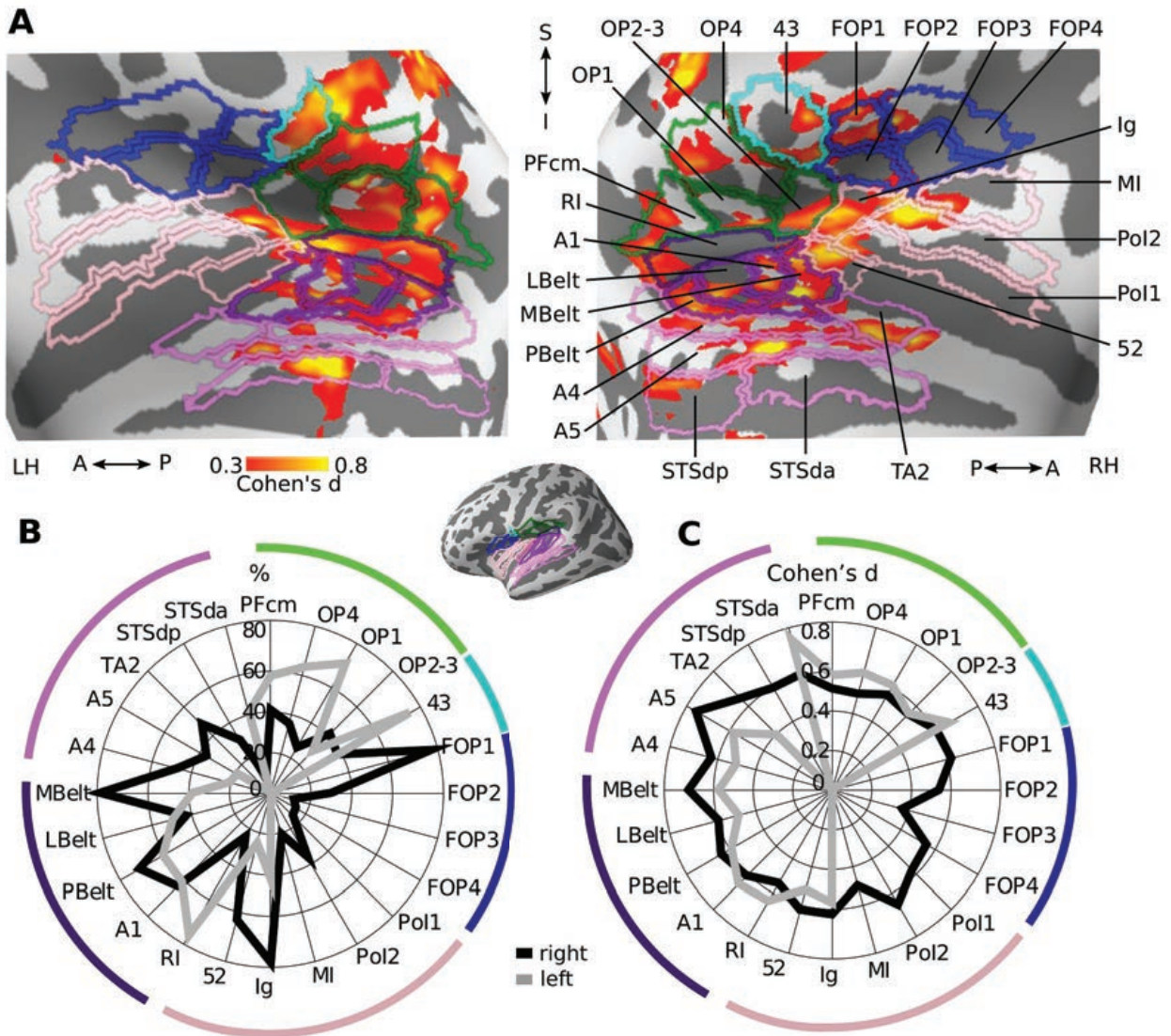

Percentage of region in gastric network

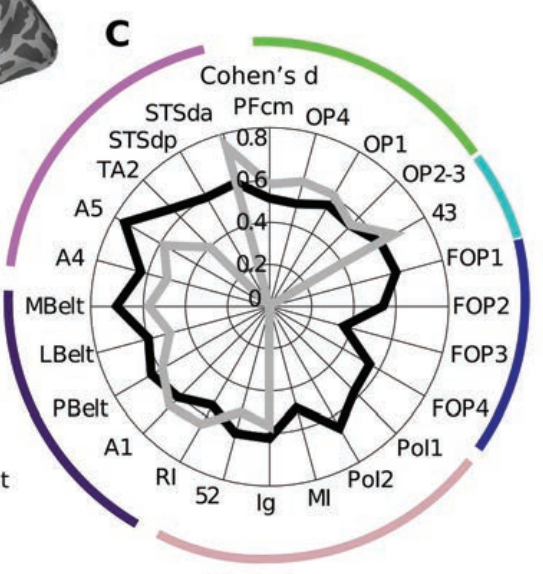

Effect sizes 

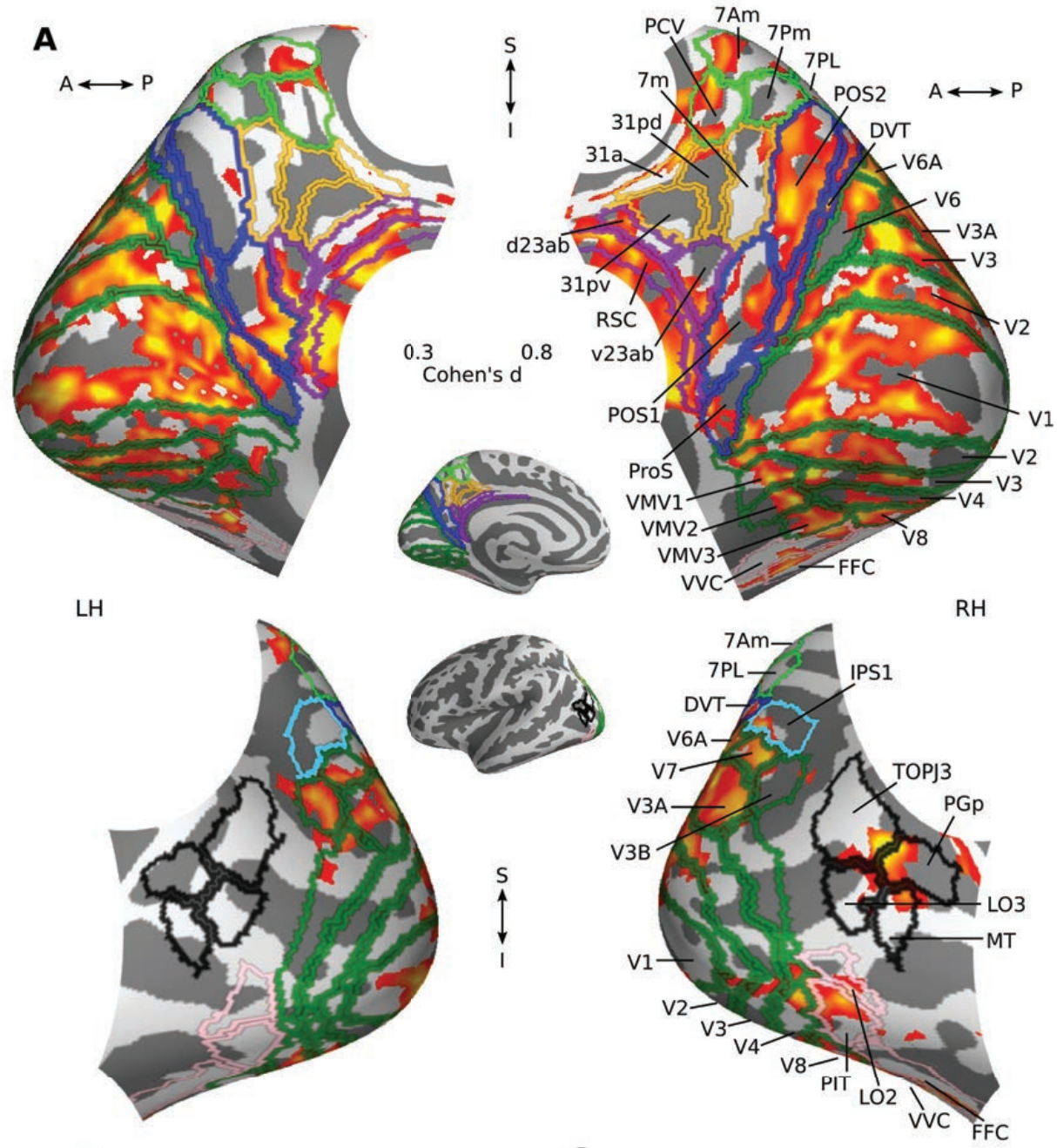

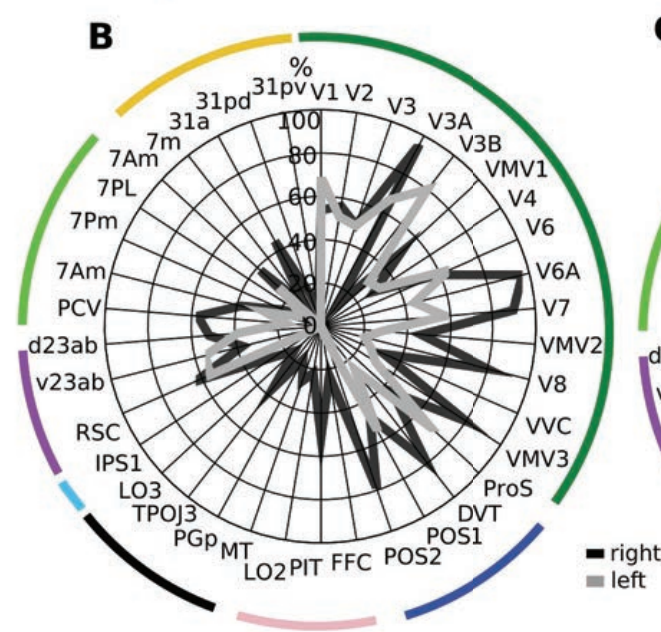

Percentage of region in gastric network
C

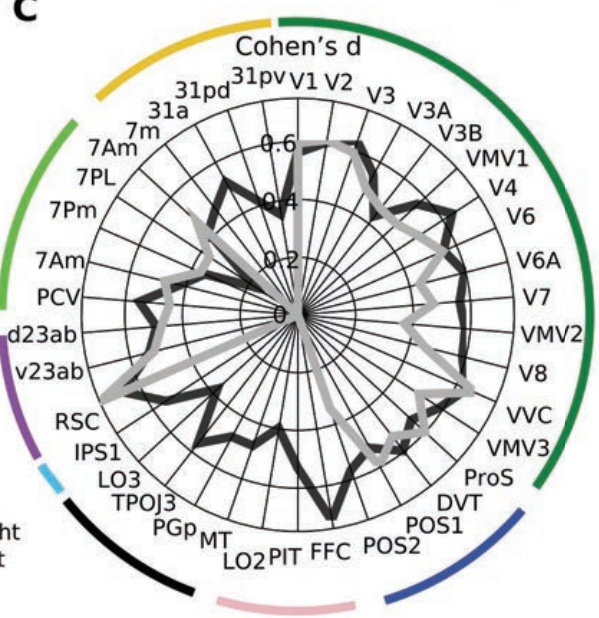

Effect sizes (Cohen's d) 


\section{A}
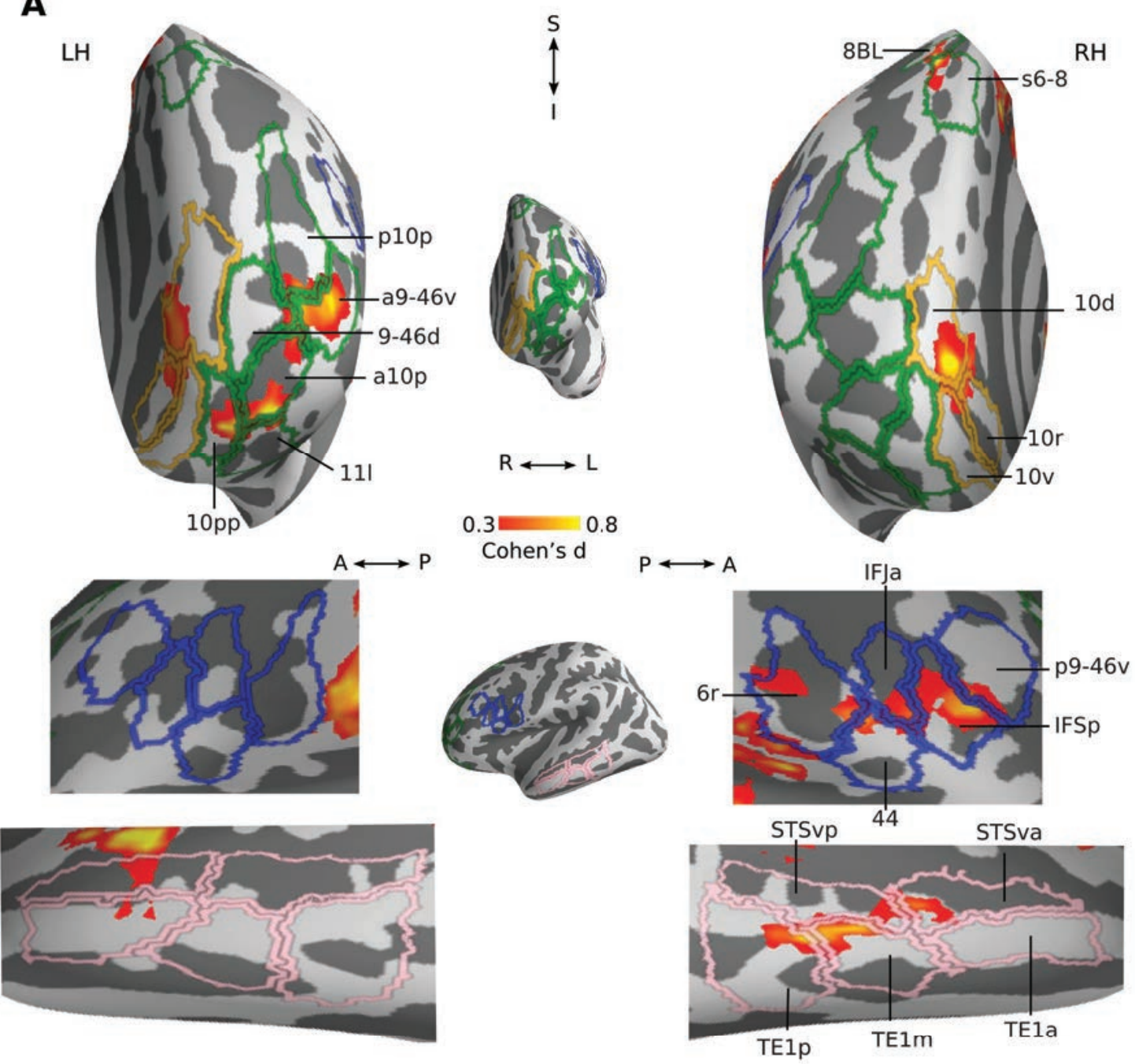

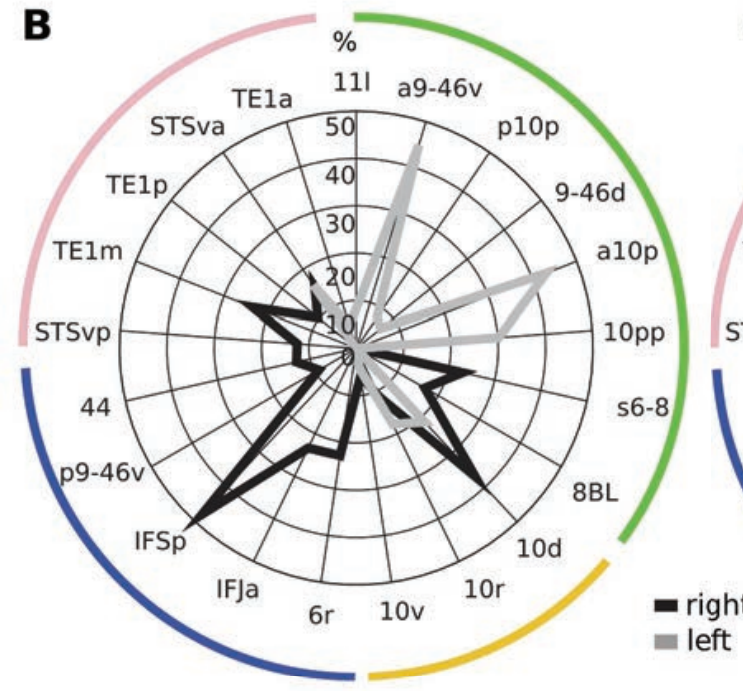

Percentage of region in gastric network

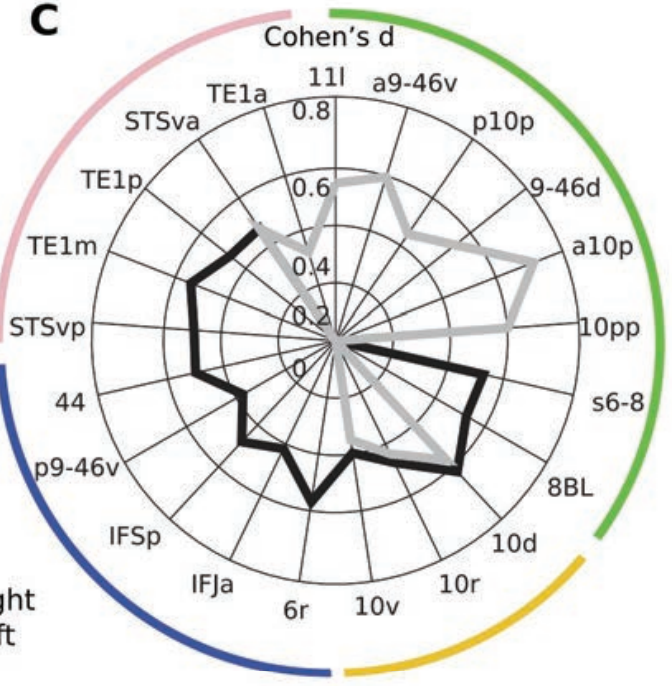

Effect Sizes (Cohen's d) 


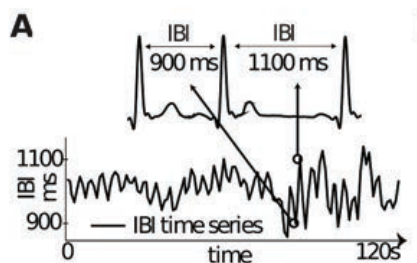

c
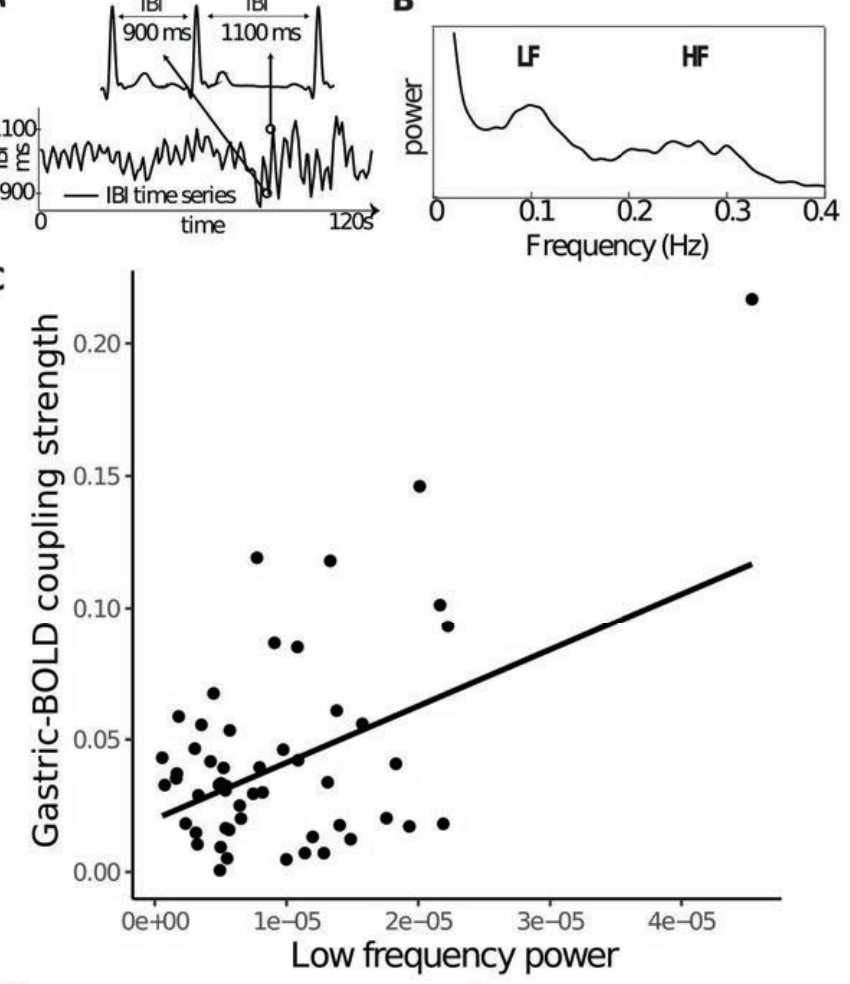

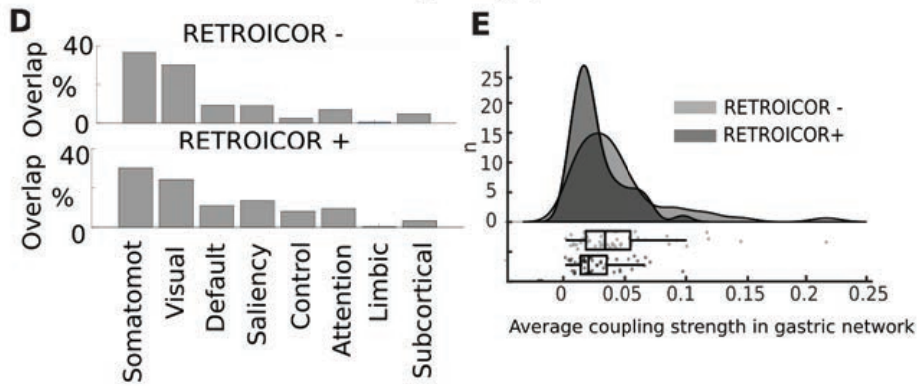

